\title{
SEARCHES FOR HIGH-ENERGY NEUTRINO EMISSION IN THE GALAXY WITH THE COMBINED ICECUBE-AMANDA DETECTOR
}

(IceCube Collaboration)

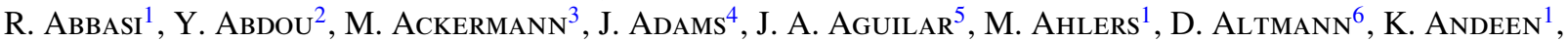

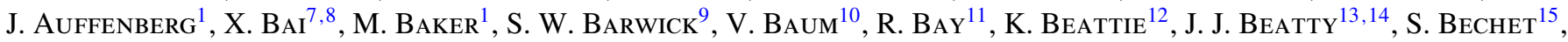
J. Becker Tuus ${ }^{16}$, K.-H. Becker ${ }^{17}$, M. Bell ${ }^{18}$, M. L. Benabderrahmane ${ }^{3}$, S. BenZvi ${ }^{1}$, J. BerdermanN ${ }^{3}$, P. Berghaus ${ }^{3}$, D. Berley ${ }^{19}$, E. Bernardini ${ }^{3}$, D. Bertrand ${ }^{15}$, D. Z. Besson ${ }^{20}$, D. Bindig ${ }^{17}$, M. BissoK ${ }^{21}$, E. Blaufuss ${ }^{19}$, J. Blumenthal ${ }^{21}$, D. J. Boersma ${ }^{21}$, C. Bohm ${ }^{22}$, D. Bose ${ }^{23}$, S. BÖser ${ }^{24}$, O. BotNer ${ }^{25}$, L. BraYeur ${ }^{23}$, A. M. Brown ${ }^{4}$, R. BruiJn ${ }^{26}$, J. BrunNer ${ }^{3}$, S. Buitink ${ }^{23}$, M. Carson ${ }^{2}$, J. Casey ${ }^{27}$, M. Casier ${ }^{23}$, D. Chirkin ${ }^{1}$, B. Christy ${ }^{19}$, F. Clevermann ${ }^{28}$, S. Cohen ${ }^{26}$, D. F. Cowen ${ }^{18,29}$, A. H. Cruz Silva ${ }^{3}$, M. Danninger ${ }^{22}$, J. Daughhetee ${ }^{27}$, J. C. Davis ${ }^{13}$, C. De ClercQ ${ }^{23}$, F. Descamps ${ }^{1}$, P. Desiati ${ }^{1}$, G. De Vries-Uiterweerd ${ }^{2}$, T. DeYoung ${ }^{18}$, J. C. Díaz-Vélez ${ }^{1}$, J. Dreyer ${ }^{16}$, J. P. Dumm ${ }^{1}$, M. Dunkman ${ }^{18}$,

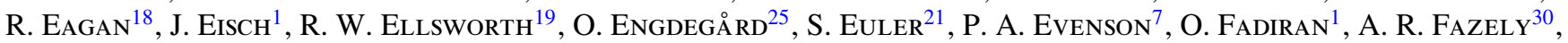
A. Fedynitch ${ }^{16}$, J. Feintzeig ${ }^{1}$, T. Feusels ${ }^{2}$, K. Filimonov ${ }^{11}$, C. Finley $^{22}$, T. Fischer-Wasels ${ }^{17}$, S. Flis ${ }^{22}$, A. Franckowiak ${ }^{24}$, R. Franke ${ }^{3}$, K. Frantzen ${ }^{28}$, T. Fuchs ${ }^{28}$, T. K. Gaisser ${ }^{7}$, J. Gallagher ${ }^{31}$, L. Gerhardt ${ }^{11,12}$, L. Gladstone ${ }^{1}$, T. GlüsenkamP ${ }^{3}$, A. GoldschmidT ${ }^{12}$, J. A. Goodman ${ }^{19}$, D. GórA ${ }^{3}$, D. Grant ${ }^{32}$, A. Groß ${ }^{33}$, S. Grullon ${ }^{1}$, M. GurTner ${ }^{17}$, C. HA ${ }^{11,12}$,

A. Haj Ismail ${ }^{2}$, A. Hallgren ${ }^{25}$, F. Halzen ${ }^{1}$, K. Hanson ${ }^{15}$, D. Heereman ${ }^{15}$, P. Heimann ${ }^{21}$, D. Heinen ${ }^{21}$, K. Helbing ${ }^{17}$,

R. Hellauer ${ }^{19}$, S. HickFord ${ }^{4}$, G. C. Hill ${ }^{34}$, K. D. HofFMAN ${ }^{19}$, R. HofFMANN ${ }^{17}$, A. Homeier ${ }^{24}$, K. Hoshina ${ }^{1}$, W. Huelsnitz ${ }^{19,35}$, P. O. Hulth ${ }^{22}$, K. Hultevist ${ }^{22}$, S. Hussain ${ }^{7}$, A. Ishihara ${ }^{36}$, E. JACOBI ${ }^{3}$, J. JacobSen ${ }^{1}$, G. S. JAPARIDZE ${ }^{37}$, O. Jlelati ${ }^{2}$, A. Kappes ${ }^{6}$, T. Karg ${ }^{3}$, A. Karle ${ }^{1}$, J. Kiryluk ${ }^{38}$, F. Kislat ${ }^{3}$, J. Klä̈s ${ }^{17}$, S. R. KLein ${ }^{11,12}$, J.-H. Köhne ${ }^{28}$, G. Kohnen ${ }^{39}$, H. Kolanoski ${ }^{6}$, L. KöPKe ${ }^{10}$, C. KopPer ${ }^{1}$, S. KopPer ${ }^{17}$, D. J. KosKinen ${ }^{18}$, M. KowalsKi ${ }^{24}$, M. Krasberg ${ }^{1}$, G. Kroll ${ }^{10}$, J. Kunnen ${ }^{23}$, N. Kurahashi ${ }^{1}$, T. Kuwabara ${ }^{7}$, M. Labare ${ }^{23}$, K. Laihem ${ }^{21}$, H. Landsman ${ }^{1}$, M. J. Larson ${ }^{40}$,

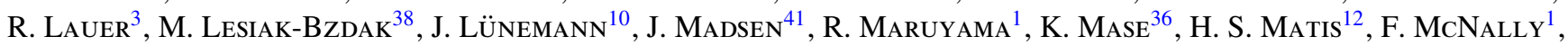
K. Meagher ${ }^{19}$, M. MercK ${ }^{1}$, P. Mészáros ${ }^{18,29}$, T. Meures ${ }^{15}$, S. Miarecki ${ }^{11,12}$, E. Middell $^{3}$, N. Milke ${ }^{28}$, J. Miller $^{23}$, L. Mohrmann ${ }^{3}$, T. Montaruli ${ }^{5,42}$, R. Morse ${ }^{1}$, S. M. Movit ${ }^{29}$, R. NahnhaUeR ${ }^{3}$, U. Naumann ${ }^{17}$, S. C. Nowicki ${ }^{32}$,

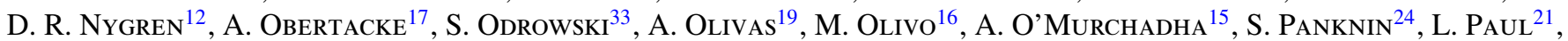
J. A. Pepper ${ }^{40}$, C. Pérez de los Heros ${ }^{25}$, D. Pieloth ${ }^{28}$, N. Pirk ${ }^{3}$, J. Posselt ${ }^{17}$, P. B. Price ${ }^{11}$, G. T. Przybylski ${ }^{12}$, L. Rädel ${ }^{21}$,

K. RAWLins ${ }^{43}$, P. RedL ${ }^{19}$, E. Resconi ${ }^{33}$, W. RhODE ${ }^{28}$, M. Ribordy ${ }^{26}$, M. Richman ${ }^{19}$, B. RIEdeL ${ }^{1}$, J. P. Rodrigues ${ }^{1}$,

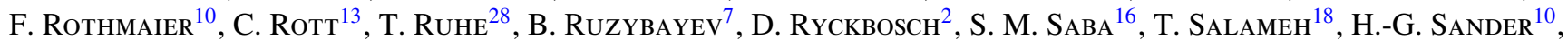
M. Santander ${ }^{1}$, S. Sarkar ${ }^{44}$, K. Schatto ${ }^{10}$, M. Scheel ${ }^{21}$, F. Scheriad ${ }^{28}$, T. SchmidT ${ }^{19}$, M. Schmitz ${ }^{28}$, S. Schoenen ${ }^{21}$, S. Schöneberg ${ }^{16}$, L. SchönherR ${ }^{21}$, A. Schönwald ${ }^{3}$, A. Schukraft ${ }^{21}$, L. Schulte ${ }^{24}$, O. Schulz ${ }^{33}$, D. SeCKel ${ }^{7}$, S. H. SeO ${ }^{22}$, Y. Sesta yo ${ }^{33}$, S. Seunarine ${ }^{45}$, M. W. E. Smith ${ }^{18}$, M. Soiron ${ }^{21}$, D. Soldin ${ }^{17}$, G. M. SPiczak ${ }^{41}$, C. Spiering ${ }^{3}$,

M. Stamatikos ${ }^{13,46}$, T. Stanev $^{7}$, A. StasiK ${ }^{24}$, T. Stezelberger ${ }^{12}$, R. G. Stokstad ${ }^{12}$, A. StößL ${ }^{3}$, E. A. StrahleR ${ }^{23}$, R. Ström ${ }^{25}$, G. W. Sullivan ${ }^{19}$, H. TAavola ${ }^{25}$, I. Taboada ${ }^{27}$, A. Tamburro ${ }^{7}$, S. Ter-Antonyan ${ }^{30}$, S. Tilav $^{7}$, P. A. Toale ${ }^{40}$, S. Toscano ${ }^{1}$, M. Usner ${ }^{24}$, D. van der Drift ${ }^{11,12}$, N. van EIJNdhoven ${ }^{23}$, A. VAN Overloop ${ }^{2}$, J. van SANTEN ${ }^{1}$, M. Vehring ${ }^{21}$, M. Voge ${ }^{24}$, C. Walck ${ }^{22}$, T. Waldenmaier ${ }^{6}$, M. WallrafF $^{21}$, M. Walter $^{3}$, R. Wasserman ${ }^{18}$, Ch. Weaver ${ }^{1}$, C. Wendi $^{1}$,

S. Westerhoff ${ }^{1}$, N. Whitehorn ${ }^{1}$, K. Wiebe ${ }^{10}$, C. H. Wiebusch ${ }^{21}$, D. R. Williams ${ }^{40}$, H. Wissing ${ }^{19}$, M. Wolf ${ }^{22}$,

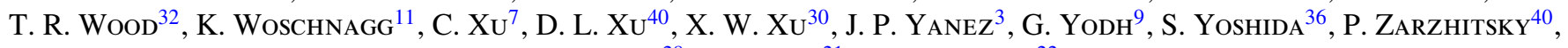
J. ZIEMANN ${ }^{28}$, A. ZILLES ${ }^{21}$, AND M. ZOLL ${ }^{22}$

${ }^{1}$ Department of Physics and Wisconsin IceCube Particle Astrophysics Center, University of Wisconsin, Madison, WI 53706, USA

${ }^{2}$ Department of Physics and Astronomy, University of Gent, B-9000 Gent, Belgium ${ }^{3}$ DESY, D-15735 Zeuthen, Germany

${ }^{4}$ Department of Physics and Astronomy, University of Canterbury, Private Bag 4800, Christchurch, New Zealand

${ }^{5}$ Département de physique nucléaire et corpusculaire, Université de Genève, CH-1211 Genève, Switzerland

${ }^{6}$ Institut für Physik, Humboldt-Universität zu Berlin, D-12489 Berlin, Germany

${ }^{7}$ Bartol Research Institute and Department of Physics and Astronomy, University of Delaware, Newark, DE 19716, USA

${ }^{8}$ Physics Department, South Dakota School of Mines and Technology, Rapid City, SD 57701, USA

${ }^{9}$ Department of Physics and Astronomy, University of California, Irvine, CA 92697, USA

${ }^{10}$ Institute of Physics, University of Mainz, Staudinger Weg 7, D-55099 Mainz, Germany

${ }^{11}$ Department of Physics, University of California, Berkeley, CA 94720, USA

${ }^{12}$ Lawrence Berkeley National Laboratory, Berkeley, CA 94720, USA

${ }^{13}$ Department of Physics and Center for Cosmology and Astro-Particle Physics, Ohio State University, Columbus, OH 43210, USA

${ }^{14}$ Department of Astronomy, Ohio State University, Columbus, OH 43210, USA

${ }^{15}$ Université Libre de Bruxelles, Science Faculty CP230, B-1050 Brussels, Belgium

${ }^{16}$ Fakultät für Physik \& Astronomie, Ruhr-Universität Bochum, D-44780 Bochum, Germany

${ }^{17}$ Department of Physics, University of Wuppertal, D-42119 Wuppertal, Germany

${ }^{18}$ Department of Physics, Pennsylvania State University, University Park, PA 16802, USA

${ }^{19}$ Department of Physics, University of Maryland, College Park, MD 20742, USA

${ }^{20}$ Department of Physics and Astronomy, University of Kansas, Lawrence, KS 66045, USA

${ }^{21}$ III. Physikalisches Institut, RWTH Aachen University, D-52056 Aachen, Germany

22 Oskar Klein Centre and Department of Physics, Stockholm University, SE-10691 Stockholm, Sweden 


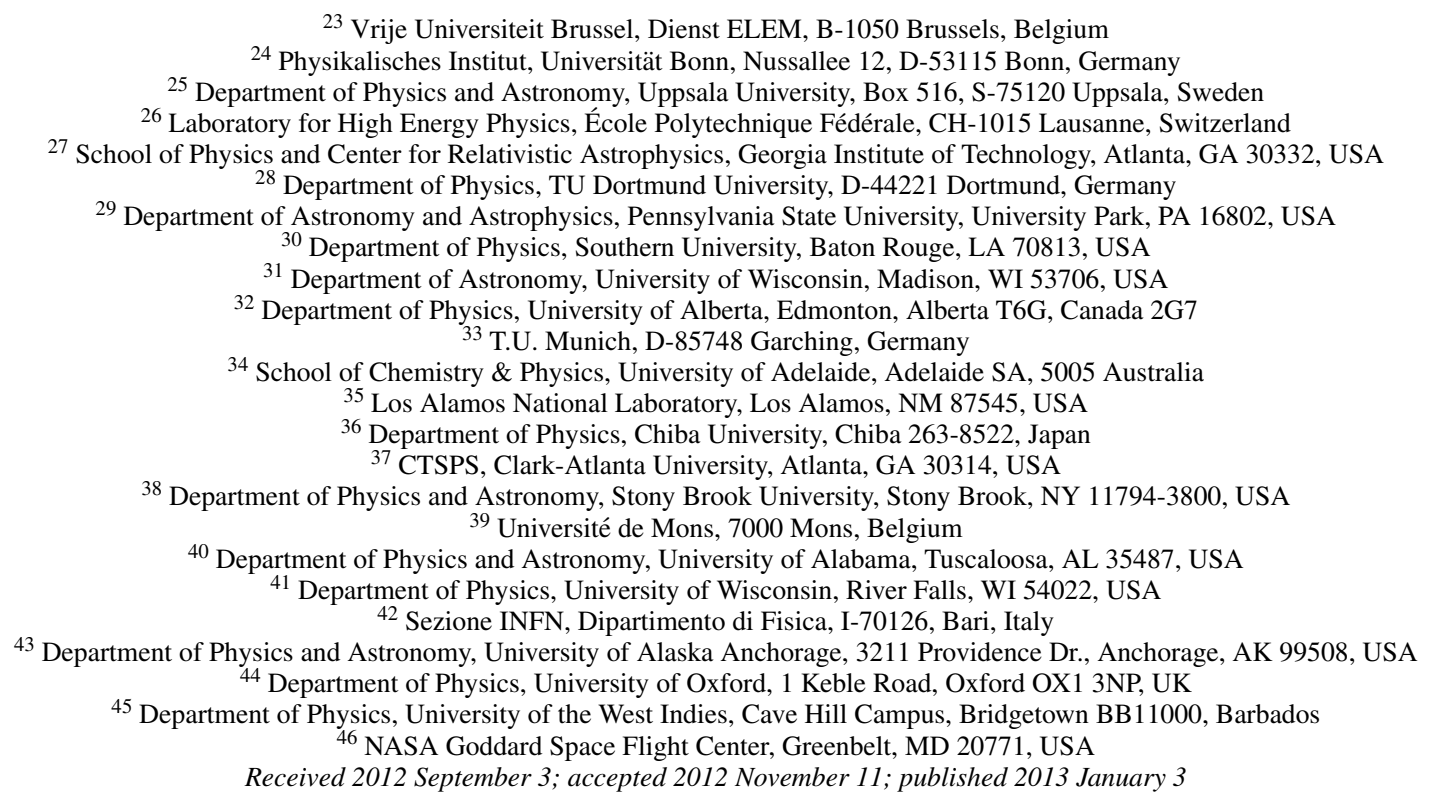

\section{ABSTRACT}

We report on searches for neutrino sources at energies above $200 \mathrm{GeV}$ in the Northern sky of the Galactic plane, using the data collected by the South Pole neutrino telescope, IceCube, and AMANDA. The Galactic region considered in this work includes the local arm toward the Cygnus region and our closest approach to the Perseus Arm. The searches are based on the data collected between 2007 and 2009. During this time AMANDA was an integrated part of IceCube, which was still under construction and operated with 22 strings (2007-2008) and 40 strings (2008-2009) of optical modules deployed in the ice. By combining the advantages of the larger IceCube detector with the lower energy threshold of the more compact AMANDA detector, we obtain an improved sensitivity at energies below $\sim 10 \mathrm{TeV}$ with respect to previous searches. The analyses presented here are a scan for point sources within the Galactic plane, a search optimized for multiple and extended sources in the Cygnus region, which might be below the sensitivity of the point source scan, and studies of seven pre-selected neutrino source candidates. For one of them, Cygnus X-3, a time-dependent search for neutrino emission in coincidence with observed radio and X-ray flares has been performed. No evidence of a signal is found, and upper limits are reported for each of the searches. We investigate neutrino spectra proportional to $E^{-2}$ and $E^{-3}$ in order to cover the entire range of possible neutrino spectra. The steeply falling $E^{-3}$ neutrino spectrum can also be used to approximate neutrino energy spectra with energy cutoffs below $50 \mathrm{TeV}$ since these result in a similar energy distribution of events in the detector. For the region of the Galactic plane visible in the Northern sky, the $90 \%$ confidence level muon neutrino flux upper limits are in the range $E^{3} d N / d E \sim 5.4-19.5 \times 10^{-11} \mathrm{TeV}^{2} \mathrm{~cm}^{-2} \mathrm{~s}^{-1}$ for point-like neutrino sources in the energy region $[180.0 \mathrm{GeV}-20.5 \mathrm{TeV}]$. These represent the most stringent upper limits for soft-spectra neutrino sources within the Galaxy reported to date.

Key words: acceleration of particles - cosmic rays - neutrinos

Online-only material: color figure

\section{INTRODUCTION}

The IceCube neutrino telescope at the South Pole was successfully completed in 2010 December. IceCube is the most sensitive telescope to date that searches for high-energy neutrino sources, whose existence is intimately related to the acceleration of hadrons and their interaction in the environment of their accelerator. The interaction of high-energy protons and nuclei with ambient matter or radiation leads to the generation of both gamma-rays and neutrinos of similar energy (Kelner et al. 2006; Kelner \& Aharonian 2008). However, it is difficult to infer the contribution of a possible hadronic component from the observed gamma-rays, since gammaray emission can also be produced by relativistic electrons via inverse Compton scattering. Moreover, the most energetic gamma-rays have a high probability of being absorbed on their way to Earth, and after successive absorption and emission processes (Moskalenko et al. 2006), the observed spectra may not be the same as the primary spectra. The detection of a flux of high-energy neutrinos from astrophysical sources, even if challenging, can thus provide unique insights into the acceleration mechanisms and the origin of cosmic rays.

The IceCube neutrino telescope has a full-sky field of view at any time and thus has the potential to observe neutrino point sources at any position in the sky albeit with different discovery potential depending on the source location and the neutrino energy spectrum of the source. For a source following an $E^{-2}$ spectrum in the energy range from $1 \mathrm{TeV}$ up to a few $\mathrm{PeV}$, IceCube can discover high-energy neutrino sources at the flux level of $10^{-11}$ to $10^{-12} \mathrm{erg} \mathrm{cm}^{-2} \mathrm{~s}^{-1}$ (Abbasi et al. 2011a) if the 
source location is known from other observations. Assuming isotropic emission, this translates to source luminosities of $L(E>1 \mathrm{TeV}) \sim 10^{33} \mathrm{erg} \mathrm{s}^{-1}$ for a source at a distance of $2 \mathrm{kpc}$. A search for neutrino point sources at any location in the sky with the IceCube 40 string detector has been presented in Abbasi et al. (2011a) using $E^{-2}$ and flatter spectra for the optimization of the analysis. This work focuses on the more specific case of Galactic neutrino sources and the energy spectra associated with them.

Among the most promising candidate sources of cosmic rays in the Galaxy are the remnants of supernovae (both shell-type and pulsar wind nebulae), the jets of microquasars, and the collective winds of massive stars (Hillas 2005; Tavani et al. 2009; Corbel \& Fermi-LAT Collaboration 2010; Aharonian et al. 2007; Ohm et al. 2010; Marcowith et al. 2008). Due to the large amount of energy released in a supernova explosion $\left(\sim 10^{51} \mathrm{erg}\right)$, supernova remnants are prime candidates for sources of Galactic cosmic rays. In microquasars, the kinetic energy carried by the jet accounts for at least $10^{36} \mathrm{erg} \mathrm{s}^{-1}$, inferred from the observed non-thermal luminosities (Gallo et al. 2005; Margon 1984). The total energy injected into the interstellar medium by the winds of OB and Wolf-Rayet stars can be as high as $\sim 10^{39} \mathrm{erg} \mathrm{s}^{-1}$, as in the case of the Cygnus OB2 association (Lozinskaya et al. 2002) and levels of $\sim 10^{38} \mathrm{erg} \mathrm{s}^{-1}$ can be achieved by a single young pulsar. What remains undetermined is the fraction of total energy per source that goes into cosmic-ray acceleration, as well as the probability for the interaction of cosmic rays close to their source. The observation of the products of cosmic-ray interactions, i.e., gamma-rays and neutrinos, can shed light on these still unsolved problems.

The highest energies $(E>100 \mathrm{TeV})$ are only accessible by means of extensive air-shower (EAS) arrays, in the case of gamma-rays, and $\mathrm{km}^{3}$-volume neutrino detectors like IceCube. Results from Milagro (Abdo et al. 2007) and ARGO-YBJ (Bartoli et al. 2012) demonstrate that the gamma-ray emission is faint at very high energies (Borione et al. 1998). However, these gamma-ray observations do not impose constraints on neutrino production, due to internal and external absorption of gamma rays at the energies considered.

Most of our knowledge of gamma-ray sources comes from Cherenkov telescopes such as H.E.S.S., MAGIC, and VERITAS, working in the energy range $100 \mathrm{GeV}-100 \mathrm{TeV}$. In the past few years, a large family of Galactic accelerators have been observed to have the bulk of their gamma-ray emission at energies below $50 \mathrm{TeV}$ (Aharonian et al. 2006b, 2009; Albert et al. 2007c, 2007d) and/or to be softer than the $d N / d E \propto E^{-2}$ spectrum that is generally expected from firstorder Fermi shock acceleration (Fermi 1949, 1954). Many of these sources reside relatively nearby, and external gammaray absorption in the interstellar radiation field is not likely. If the detected gamma rays are related to hadronic acceleration and are produced in transparent sources, the expected distribution of neutrino energies has the same spectral index and a lower energy cutoff than the gamma-ray spectrum (Kelner et al. 2006). The modeling of cosmic-ray sources with diffusive shock acceleration also allows for the presence of spectra steeper or flatter than the generic $E^{-2}$ behavior, depending on the configuration of the shock (Bell 1978a, 1978b; Schlickeiser 1989a, 1989b; Meli et al. 2008). The influence of diffusion in the sources themselves may modify the spectra to produce primary spectra of $E^{-2.3}$ or steeper (Biermann et al. 2009, 2010). In order to target soft-spectra sources, we have optimized the search reported here for a generic spectrum proportional to $E^{-3}$.

In this work, we use the 22 and 40 string configurations of IceCube (IC22, IC40) as well as the Antarctic Muon And Neutrino Detector Array (AMANDA, IC22+A, IC40+A) to enhance the sensitivity for soft-spectra sources, or sources with energy cutoffs. We have used AMANDA as an integrated low-energy extension of IC22 and IC40 and developed an analysis strategy that is optimized for a high retention of signal events below $10 \mathrm{TeV}$. We have used the resulting low-energy optimized data samples to search for Galactic neutrino emission above $\approx 200 \mathrm{GeV}$. At these energies, IceCube's field of view covers the range of Galactic longitude $40^{\circ}<\ell<210^{\circ}$, as illustrated in Figure 1. The searches performed include a scan of the accessible part of the Galactic plane, a dedicated analysis of the Cygnus region, the search for neutrino emission from a pre-defined list of interesting astrophysical objects, and an analysis that searches for time-dependent neutrino emission from Cygnus X-3 in correlation with radio flares. The paper is organized as follows. Section 2 describes the relevant technical aspects of IceCube, AMANDA, and the integration of AMANDA into IceCube. Section 3 reports the analysis methods that have been applied and the respective astrophysical targets. Section 4 explains the details and characteristics of the data samples obtained, and Section 5 provides the results.

\section{THE COMBINED DETECTOR: ICECUBE AND AMANDA}

\subsection{IceCube}

During the construction phase from 2004 to 2010, the operational configuration of IceCube increased year by year (see Figure 2) to finally cover a volume of approximately $1 \mathrm{~km}^{3}$. IceCube, including its DeepCore extension, is composed of 86 strings each holding 60 digital optical modules (DOMs). Each DOM contains a 10 inch photomultiplier tube (PMT) and an onboard signal read-out and digitization system, all housed in a glass pressure vessel (Abbasi et al. 2009b). Of the 86 strings in the array, 78 form a hexagonal grid with a typical distance of $125 \mathrm{~m}$ between neighboring strings. The vertical distance between DOMs on the same string is $17 \mathrm{~m}$. The remaining eight strings are part of the low-energy extension DeepCore (Abbasi et al. 2012b) and are deployed in the deepest, clearest ice at the center of the detector with a smaller vertical and horizontal spacing between the DOMs of $7 \mathrm{~m}$ and $60 \mathrm{~m}$, respectively. The DOMs detect Cherenkov radiation emitted by secondary charged particles produced in interactions of high-energy neutrinos with nuclei in the ice or the bedrock below the ice. To enhance the detection of light from upwardgoing particles, the PMTs point downward. In order to avoid a deterioration of the analog PMT signal, the signal is digitized directly in the DOMs with a set of analog transient waveform digitizers and a fast analog to digital converter (fADC; Abbasi et al. 2010). The events that are used in this analysis are selected by a multiplicity trigger which requires at least eight hit DOMs within a time window of $5 \mu \mathrm{s}$. The DOMs send their recorded signals to the surface and an event is constructed if the trigger conditions are met. In the detector configurations used in this work, only DOMs for which there is also a signal from one of the nearest two DOMs above or the nearest two DOMs below within $1 \mu \mathrm{s}$ (so-called hard local coincidence) are considered in the trigger and the event building to suppress noise contributions. An event contains all DOM readouts associated with the trigger 


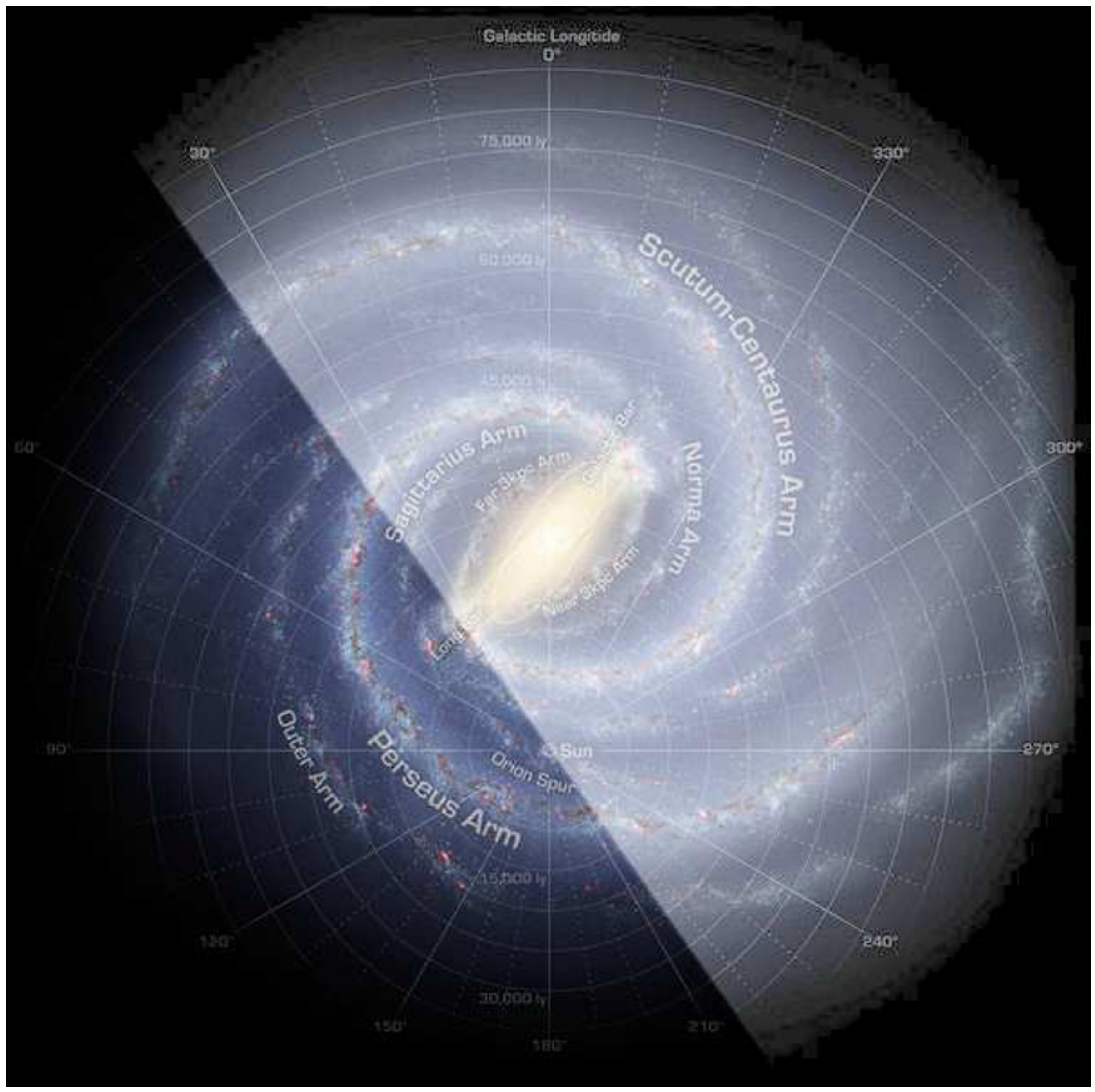

Figure 1. Artistic rendering of the Milky Way made from optical, IR, and radio data from Churchwell et al. (2009). The part of the Galaxy within the field of view of the IceCube analyses in this paper is from Galactic longitude $40^{\circ}<\ell<210^{\circ}$ (i.e., lower left region).

(A color version of this figure is available in the online journal.)

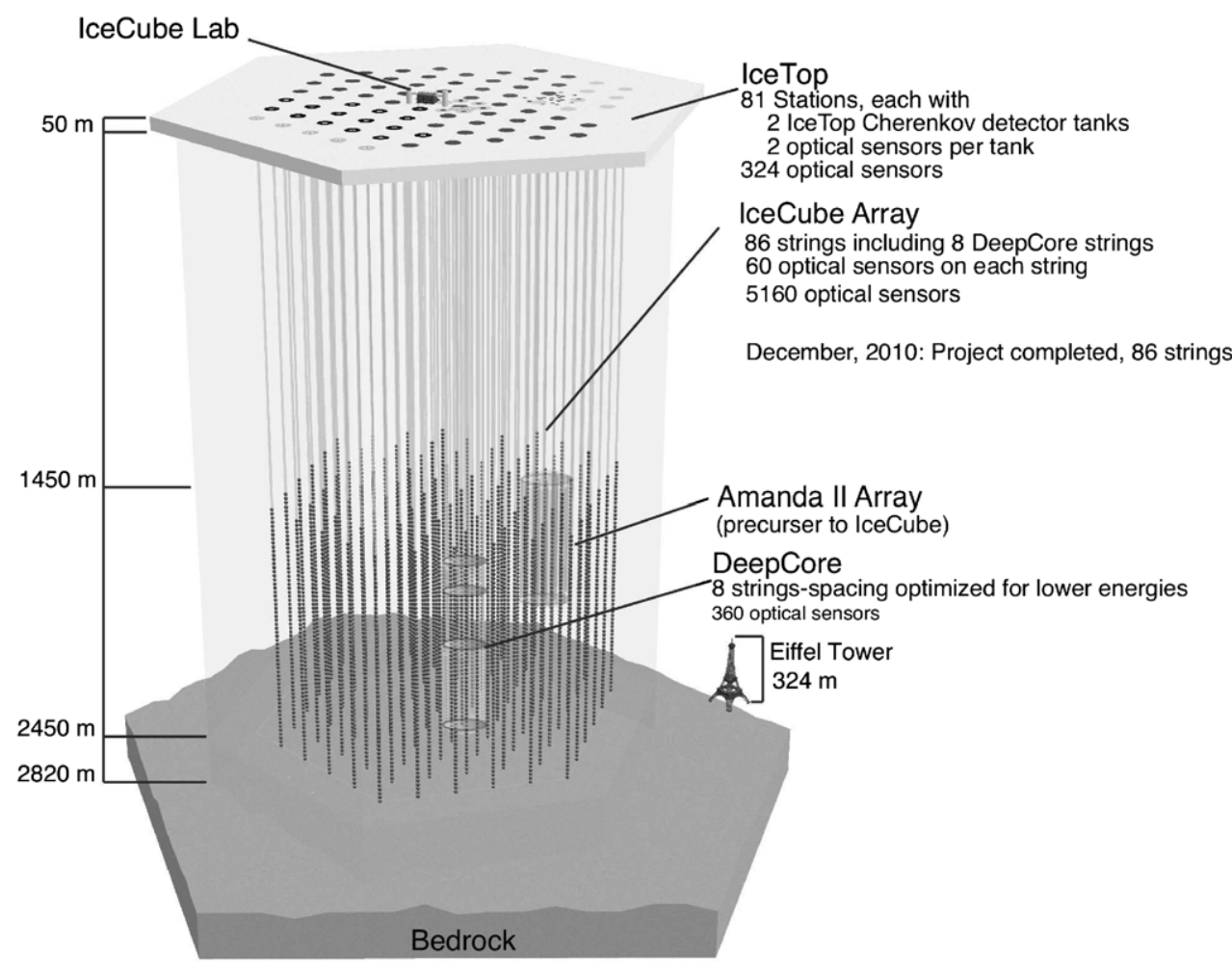

Figure 2. View of the IceCube array. AMANDA is completely surrounded by IceCube strings and presents a more compact structure. 
as well as all further readouts within $\pm 10 \mu$ s around the trigger time.

\subsection{AMANDA}

After a construction phase from 1993 until 2000, the completed AMANDA-II detector took data as a stand-alone neutrino telescope from 2000 February until 2006 December. This configuration consisted of 677 optical modules (OMs) on 19 strings. Most of the optical modules were deployed at depths between 1500 and $2000 \mathrm{~m}$ whereas IceCube extends down to $2450 \mathrm{~m}$. For data analysis, a total of 526 OMs have been used. The AMANDA strings follow a roughly cylindrical geometry, as is shown in Figure 2.

The typical distance between adjacent strings is around $40 \mathrm{~m}$ and the average vertical spacing between the modules is about 15 m. From 2007 February until 2009 April, AMANDA was operated as an integrated part of IceCube. In many respects, IceCube is technologically more advanced than AMANDA, reflecting general progress as well as experience collected during the operation of AMANDA (Ackermann et al. 2006). In particular, the signal transfer from the optical modules to the surface is different. As mentioned above, IceCube DOMs digitize the PMT signal directly in the ice. They also generate the $\mathrm{HV}$ for the PMTs in the DOMs. In contrast, AMANDA OMs produced analog signals that were sent to the AMANDA data acquisition system which was located in the Martin A. Pomerantz Observatory (MAPO). The original data acquisition system (DAQ) could register the leading and trailing edge time of up to 8 pulses per OM per event and only the total charge. The same cables were used to transfer the analog data and to provide the HV to the PMTs. For AMANDA strings 11-19, an additional connection via optical fibers was installed to transmit the PMT signals with a better time resolution. Moreover, AMANDA string 18 (Ackermann et al. 2006) was equipped with prototypes for the IceCube DOMs, including the capability of onboard waveform digitization. This option, however, was used only in testing mode and was not included in the data acquisition schemes used for physics analysis.

The AMANDA DAQ was upgraded starting from 2002. Flash ADC modules called transient waveform recorders (TWRs) were installed in the new DAQ in order to digitize the analog waveforms from the AMANDA OMs at the surface. The upgraded data acquisition operated parallel to the analog one until 2006. From 2007 on, only the TWR-DAQ was operational. This surface waveform digitization stored more information such as the induced charge and the arrival times of individual pulses. As the TWR-DAQ was also faster than the previous DAQ, the trigger threshold could be reduced. Trigger thresholds from 8 to 13 hit OMs were used during different years. This upgrade significantly improved the performance of AMANDA and ultimately allowed us to use it to enhance IceCube's performance at low energies (Abbasi et al. 2012a).

\subsection{AMANDA as an Integrated Part of IceCube}

Since AMANDA is about eight times more densely instrumented than IceCube and fully surrounded by IceCube strings, it offered the potential to increase the low-energy performance of IceCube and to be used as the first low-energy core inside a large neutrino telescope. This led to its integration into the data taking of IceCube.

The operational integration of AMANDA into IceCube required the establishment of connections between the two detectors for the exchange of trigger information to be able to merge events as well as for an accurate synchronization in time. MAPO is about $300 \mathrm{~m}$ away from the IceCube Control Lab (ICL), which houses the IceCube surface data acquisition. Optical fibers have been used in order to connect the two buildings. Moreover, a TCP/IP connection was established for the communication between the buildings. A GPS module was installed to synchronize the TWRs responsible for the digitization of the AMANDA waveforms and to synchronize the detector with IceCube. The IceCube clock was used as a reference. An optical fiber connection was used to transmit the AMANDA trigger signal to IceCube. In the integrated mode, AMANDA and IceCube were still triggered separately. Since AMANDA had a lower energy threshold than IceCube, a readout of IceCube was initiated every time AMANDA triggered, even if there were not sufficient hits in IceCube to produce a trigger by itself. AMANDA was not read out in correspondence with IceCube triggers. Events from AMANDA and IceCube were merged on the basis of a time coincidence. Because the duration of AMANDA records was fixed to $10.24 \mu \mathrm{s}$, while the duration of IceCube records was extended if new triggers occurred within the read-out window, there was a possibility that more than a single record in AMANDA was associated with a single record in IceCube. In this case, they were all included in the same combined event.

\section{METHODS AND TARGETS}

The IceCube neutrino telescope monitors the entire sky without the need for explicit pointing. The energy- and zenithdependent sensitivity of the IceCube 40 string configuration are described in Abbasi et al. (2011a). In previous works, a generic, unbroken $d N / d E \propto E^{-2}$ signal spectrum up to the PeV region has been assumed for the optimization of the data analysis and the evaluation of the detector performance (Abbasi et al. 2011a). This approach achieves the best signal-to-noise in the energy range above a few $\mathrm{TeV}$, since the assumed signal spectrum is significantly harder than the characteristic spectrum of (background) atmospheric neutrinos $d N / d E \propto E^{-3.7}$. However, a lower energy threshold is of primary importance for the search for neutrino sources characterized by an energy cutoff or by soft spectra. In the optimization of the analysis, we have used a generic, soft power-law spectrum following $d N / d E \propto E^{-3}$ and we have also considered the Crab Nebula spectrum measured by H.E.S.S. (Aharonian et al. 2006a) which would correspond to a neutrino spectrum $d N / d E \propto E^{-2.4}$ with an exponential cutoff at $7 \mathrm{TeV}$ (provided that all the measured gamma rays are of hadronic nature). This last spectrum is representative of a "low-energy" source and its study is very instructive in the understanding of the impact of an energy cutoff on the performance of IceCube. We will refer to this spectrum throughout the paper as a "Crab-like" spectrum. In the following we report on the different searches that have been performed.

\subsection{Galactic Plane Scan and Source List}

The location of the solar system in the local spiral arm gives us a particular view of the Galaxy. Given the vertical scale of the thin disk and the distribution of cold gas in the Galaxy, we see most of the galactic accelerators projected in a narrow band close to the Galactic plane. In the energy range of interest for the detection of galactic neutrino sources, IceCube can explore the Northern sky, which includes part of the first quadrant of the Galaxy, the whole second quadrant, and a small portion of the third Galactic quadrant. The search for neutrino sources in the Galactic plane is performed by superimposing 
a fine grid over the region of the sky within the Galactic coordinates $36^{\circ}<\ell<210^{\circ},-5^{\circ}<b<5^{\circ}$. The step size of the grid is chosen to be smaller than the angular resolution achieved in the analysis reported in Section 4.4.2.

In the analysis of IC22+A, a grid of $0.5 \times 0.5$ has been used, while a $0.25 \times 0.25$ grid has been chosen for the IC40+A, given its improved angular resolution (see Figure 11). At each point on the grid, an unbinned maximum likelihood ratio test is performed on all selected events. The likelihood of a composite signal and background hypothesis is compared to the background-only hypothesis, similar to the method described in Braun et al. (2008), without the inclusion of an energy term in the likelihood.

Seven particularly interesting sources have been studied individually in this analysis as representatives of different types of Galactic accelerators. The interest in these sources is motivated by the observation of a $\mathrm{GeV}-\mathrm{TeV}$ gamma-ray counterpart at the time of the analysis. For Cygnus X-3, due to the high variability in the radio and X-ray bands, we have tested the hypothesis of variable neutrino emission and performed a time-dependent analysis. The other sources are listed below and are treated as steady point-source candidates.

Crab Nebula, distance: $\approx 2.0$ kpc (Trimble 1968). The Crab Nebula is powered by a pulsar with a spin-down luminosity of $\sim 5 \times 10^{38} \mathrm{erg} \mathrm{s}^{-1}$. This energy is injected into relativistic particles and magnetic fields (Kennel \& Coroniti 1984), although the exact composition of the pulsar wind, as well as the mechanism by which the total power of the pulsar is transported and dissipated, is not known. It is an efficient particle accelerator, where $\approx 60 \%$ of the total power of the pulsar is injected into relativistic electrons which emit synchrotron radiation from radio to X-rays (Hester 2008). Although it appears as the strongest gamma-ray source in the sky, the ratio between the gamma-ray luminosity at $E>1 \mathrm{TeV}$ and the spin-down luminosity is of the order of $10^{-5}$ (Aharonian et al. 2004, 2006a). The simplest interpretation of this is that electrons rapidly lose their energy through synchrotron radiation at lower frequencies, and that the majority of cosmic rays, if present in a significant proportion, escape from the source without interaction. The constraint on the steady neutrino production in the Crab obtained by IceCube (Abbasi et al. 2011c) is at the level of $L_{v} \lesssim 2 \times 10^{35} \mathrm{erg} \mathrm{s}^{-1}$, a factor of $\approx 3.4$ larger than the luminosity in gamma rays assuming the H.E.S.S. gamma-ray spectrum (Aharonian et al. 2006a, and its corresponding neutrino spectrum expected in the case of a hadronic origin) extrapolated to the energy range between $400 \mathrm{GeV}$ and $40 \mathrm{TeV}$.

Cas A, distance: $\approx 3.4 \mathrm{kpc}$ (Reed et al. 1995). This source is a classical shell-type supernova remnant (SNR). Its high-energy gamma-ray flux was detected by HEGRA in the energy region between $1 \mathrm{TeV}$ and $10 \mathrm{TeV}$ without any evidence of an energy cutoff (Aharonian et al. 2000), and detected by MAGIC down to $250 \mathrm{GeV}$ following a power-law spectrum $\propto E^{-2.3}$ and with an integrated photon flux above $1 \mathrm{TeV}$ of $\approx 7.3 \times 10^{-13} \mathrm{~cm}^{-2} \mathrm{~s}^{-1}$ (Albert et al. 2007a).

IC 443, distance: $\approx 1.5 \mathrm{kpc}$ (Fesen 1984). IC 443 is an asymmetric shell-type SNR, where part of the shell is impacting a molecular cloud, accelerating particles to very high energies in the process. $\mathrm{TeV}$ gamma rays are observed arriving from the molecular line emission region, giving support to a hadronic origin of the $\mathrm{TeV}$ gamma rays. The spectrum measured in the energy range from $100 \mathrm{GeV}$ to $1.6 \mathrm{TeV}$ is well fitted by a very steep power-law $\propto E^{-3.1}$ (Albert et al. 2007b). The integrated photon flux above $1 \mathrm{TeV}$ obtained by extrapolation is $\approx 3.2 \times 10^{-13} \mathrm{~cm}^{-2} \mathrm{~s}^{-1}$.

W51, distance: $\approx 6.0 \mathrm{kpc}$ (Kundu \& Velusamy 1967). This source has been detected in $\mathrm{GeV}$ gamma rays by the FermiLAT telescope (Abdo et al. 2009c), at TeV energies by H.E.S.S. (Fiasson et al. 2009) and by MAGIC (Aleksić et al. 2012). The high-energy emission is thought to arise from the interaction between a composite SNR (W51C) with a molecular cloud present in the region. The high luminosities observed in $\mathrm{GeV}$ gamma rays, greater than $10^{36} \mathrm{erg} \mathrm{s}^{-1}$, and the hint of a hadronic origin for the gamma-ray spectrum make this an interesting target for IceCube despite its large distance. The MAGIC Collaboration recently extended the spectrum from the highest Fermi-LAT energies to $5 \mathrm{TeV}$ and found that the spectral index of the source follows a single power law with an index of $2.58 \pm 0.07_{\text {stat }} \pm 0.22_{\text {syst }}$ (Aleksić et al. 2012).

LS I+61 303, distance: $\approx 2.0$ kpc (Frail \& Hjellming 1991). This source is a high-mass X-ray binary with a compact object in an eccentric orbit around a Be star. The nature of the compact object is not known, and both a pulsar wind model and a microquasar model have been suggested for this source. MAGIC detected very high energy emission modulated with the orbital period (Jogler et al. 2008). The highest significant detection is obtained around apastron, at orbital phases $0.6-0.7$, with a spectrum following $d N / d E \approx 2.6 \times 10^{-12} E^{-2.6} \mathrm{TeV}^{-1} \mathrm{~cm}^{-2} \mathrm{~s}^{-1}$, at $E>300 \mathrm{GeV}$. No TeV emission is observed at periastron, although significant gamma-ray absorption in the strong radiation field of the Be star is expected in this case (SierpowskaBartosik et al. 2009). This scenario is supported by the detection of MeV-GeV gamma rays by the Fermi satellite (Abdo et al. 2009b), which may result from the cascade process in $\gamma \gamma \rightarrow e^{+} e^{-}$. The hypothesis of particle injection along the whole orbit is then a plausible option. This, together with the considerable amount of both matter and radiation from the companion star available for cosmic-ray interactions, makes this source an interesting candidate for steady neutrino emission. For the search for periodic neutrino emission from binary systems performed by IceCube, refer to Abbasi et al. (2011b).

SS 433, distance: $\approx 5.0 \mathrm{kpc}$ (Romney et al. 1987). SS 433 is a confirmed microquasar and a black hole candidate in orbit around a massive star. The source exhibits two oppositely directed relativistic jets which are thought to eject material at a rate larger than $10^{-6} M_{\odot} \mathrm{yr}^{-1}$ (Begelman et al. 1980). It is the only X-ray binary system in which hadrons have been found in the jet (Migliari et al. 2002). The entire source is embedded within a nebulous structure (W50) which is thought to be the expanding supernova shells of the progenitor star of the black hole in SS 433. The source has been searched for by the HEGRA, MAGIC, and CANGAROO-II Cherenkov telescopes (Aharonian et al. 2005; Hayashi et al. 2009), resulting in upper limits for the gamma-ray emission from both the inner system and the different interaction regions with the W50 Nebula. Strong gamma-ray absorption is expected from this system, due to the periodic eclipses through the companion star as well as attenuation due to the precession of the accretion disk envelope (Reynoso et al. 2008). As in the case of LS I+61 303, the presence of a significant amount of target material for cosmicray interactions as well as the possibility of a higher energy emission than what is inferred from gamma-ray observations due to absorption, makes this an interesting candidate for a neutrino source. SS 433 has also been tested for possible periodic neutrino emission in Abbasi et al. (2011b). 


\subsection{The Cygnus Region}

The Cygnus region is roughly located within Galactic longitudes $70^{\circ}<\ell<90^{\circ}$ and latitudes $-4^{\circ}<b<8^{\circ}$, where our line of sight is directed nearly along the local spiral arm of the Galaxy (Reipurth \& Schneider 2008). At a distance of approximately $5 \mathrm{kpc}$ our line of sight has left the local arm and crosses the Perseus arm, and even the outer arm further away $(\sim 10 \mathrm{kpc})$. Here many different sources are located at various distances superimposed in a relatively small area in the sky, resulting in a complex region which harbors some of the closest and most massive regions of star formation in the Galaxy.

The vast majority of the molecular gas detected in the Cygnus region is concentrated on the local arm (Schneider et al. 2006), at distances between 1 and $3 \mathrm{kpc}$. One of the most massive giant molecular cloud complexes in the Galaxy resides within this region, at a distance of $\approx 1.7 \mathrm{kpc}$. It is thought to be the birth place of the massive Cyg OB2 association, and probably also Cyg OB9 and Cyg OB1, as well as of a number of less massive star clusters with young stars or ongoing star formation (Le Duigou \& Knödlseder 2002). The strong stellar winds and radiation pressure of the massive stars in the Cygnus region have strongly influenced the spatial distribution of the molecular gas in the region, displacing and compressing the gas-forming filamentary structures and dense clumps which surround the less dense environment of the cluster, in which the gas has been evacuated. If high-energy particles are generated within the stellar associations, they can interact with the ultraviolet radiation fields producing $\mathrm{TeV}$ gamma-rays through the Inverse Compton and $\mathrm{p} \gamma$ processes. However, protons and nuclei can travel longer distances than electrons, and they may also leave the photondominated regions around the massive star clusters and interact with the nearby molecular clouds. The resulting neutrino flux map would then reflect the complicated distribution of the gas in the region. It is also worth noting that the injection of cosmicrays may take place at multiple locations due to the presence of several particle accelerators inside the Cygnus region. The existence of these accelerators is confirmed by the observation of strong $\mathrm{TeV}$ gamma-ray emission throughout an area of approximately $10^{\circ} \times 10^{\circ}$ (Abdo et al. 2007; Aliu et al. 2011). The potential for IceCube to observe neutrinos from this region has been discussed in Anchordoqui et al. (2006), Beacom \& Kistler (2007), and Kappes et al. (2009), based on the measured TeV gamma-ray flux.

Due to the complexity of the possible spatial distribution of events within the Cygnus region, we have applied an analysis of the spatial correlations between neutrino events to search for an astrophysical neutrino signal in an extended region. If the intensity fluctuations of a possible neutrino signal throughout the region follow a certain correlation structure, this may show up as a significant deviation from the random distribution of atmospheric neutrino events.

In the IceCube analysis, we use the two-point correlation function formalism introduced by Peebles (1980) and coworkers (Peebles \& Groth 1975; Fry \& Peebles 1978) to study the large-scale matter distribution in the universe (Madox et al. 1996). In particle astrophysics, the correlation function has been used to search for anisotropies in the spatial distribution of cosmic rays (HIRES Collaboration 2004; Finley \& Westerhoff 2004) and neutrinos (Abbasi et al. 2009c).

Here we use the approach to search for neutrinos inside an area of $11^{\circ} \times 7^{\circ}$ centered on the most active part of the Cygnus complex in $\mathrm{TeV}$ gamma rays. We define our test statistic in terms of a clustering function, $\Phi(\Theta)$, which is the excess or deficit in the number of event pairs within a certain distance with respect to the background-only hypothesis (similar to a cumulative correlation function, see, e.g., (Peebles 1980), and references therein (Kerscher et al. 2000; Landy \& Szalay 1998) for estimators of the two-point correlation function based on pair counting):

$$
\Phi(\Theta)=\frac{\int_{0}^{\Theta} D D(\Theta) d \Theta}{\int_{0}^{\Theta} R R(\Theta) d \Theta},
$$

where $\Theta$ is the distance between two events, and $D D(\Theta)=$ $\sum_{i j} D D_{i j}, R R(\Theta)=\sum_{i j} R R_{i j}$, where the sum is over all non-repeated pairs in, respectively, the real data sample and in a sample randomized in azimuth (representative of a pure background case). In our case, $D D_{i j}\left(R R_{i j}\right)=1$ only if either the event $i$ or the event $j$, or both, are within the region under study, and it is equal to zero otherwise. It is worth noting that with this definition we measure both the intensity of the process that generated the observed neutrino event pattern as well as its correlation structure.

\subsection{Cygnus $X-3$ Flares}

The high-energy sky presents strong variability in time. If neutrino emission from a particular object is expected to vary with time and if it coincides with electromagnetic emission, it is advantageous to include the time information in the data analysis. One such case is the microquasar Cygnus X-3 (Cyg $\mathrm{X}-3$ ) at a distance of $\approx 9 \mathrm{kpc}$. Coinciding with the period of data taking considered in this analysis, first observations of the production of high-energy photons within the system of Cyg X-3 were published by the Fermi (Abdo et al. 2009a) and AGILE (Tavani et al. 2009) satellite missions independently. The reported gamma-ray fluxes are in the energy band between $100 \mathrm{MeV}$ and $100 \mathrm{GeV}$ (Fermi), following a power law of spectral index $-2.70 \pm 0.25$, and between $100 \mathrm{MeV}$ and $10 \mathrm{GeV}$ (AGILE) with spectral index $-1.8 \pm 0.2$. However, gamma rays from Cyg X-3 are only observed during certain periods of time, probably correlated with strong radio outbursts and certain X-ray emission states of the system (Abdo et al. 2009a; Koljonen et al. 2010). No TeV gamma rays from Cyg X-3 have been detected by Cherenkov telescopes such as MAGIC and VERITAS so far, a possible explanation being strong absorption of high-energy photons or limited observation time. Upper limits on the integrated gamma-ray flux above $250 \mathrm{GeV}$ are at the level of $2.2 \times 10^{-12} \mathrm{~cm}^{-2} \mathrm{~s}^{-1}$ (Aleksić et al. 2010). Assuming a hadronic origin of at least part of the gamma rays, the high densities in the system of Cyg X-3 could provide an environment for copious neutrino production at $\mathrm{TeV}$ energies, detectable by neutrino telescopes such as IceCube (Abdo et al. 2009a; Bednarek 2005). A previous search for periodic neutrino emission was reported in Abbasi et al. (2011b).

Here we perform a search for neutrino emission during, or close to, the observed flaring activity in Cyg X-3. The phenomenology of emission states of Cyg X-3 has been studied carefully using radio and X-ray observations (Szostek et al. 2008; Tudose et al. 2007; Koljonen et al. 2010). With radio and $\mathrm{X}$-ray data, there are two ways to identify active periods of Cyg $\mathrm{X}-3$ associated with jet ejection: the observation of a radio flux above 1 Jy, following Szostek et al. (2008), and the observation of hyper-soft X-ray states and subsequent hardening of the $\mathrm{X}$-ray spectrum, following Koljonen et al. (2010). The identification of potential flaring periods of Cyg X-3 is thus split into 


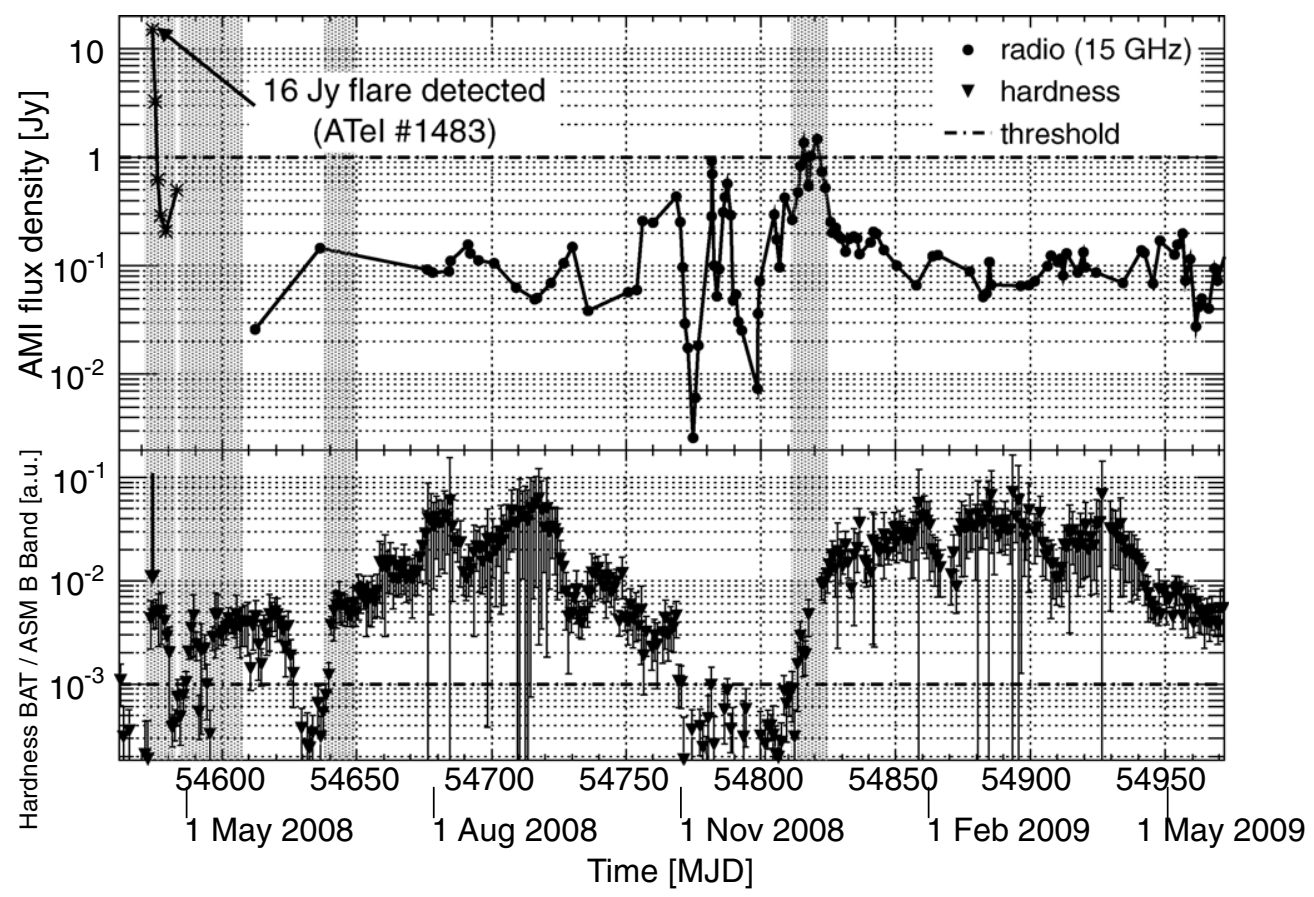

Figure 3. Radio light curve and hardness of Cyg X-3 with the four time windows of the analysis (gray shading).

Table 1

Criteria Followed for the Selection of Cyg X-3 Flaring Periods

\begin{tabular}{lccc}
\hline \hline Wavelength & Telescope & START & STOP \\
\hline Radio & AMI radio telescope (Zwart et al. 2008) & $S_{15 \mathrm{GHz}}>1 \mathrm{Jy}$ & $S_{15 \mathrm{GHz}}<1 \mathrm{Jy}^{\mathrm{a}}$ \\
X-ray & $R X T E /$ ASM (Levine et al. 1996) \& & $S_{\mathrm{fit}}>1 \mathrm{Jy}$ & $S_{\mathrm{fit}}<1 \mathrm{Jy}^{\mathrm{b}}$ \\
& Swift/BAT (Barthelmy et al. 2005) & & \\
\hline
\end{tabular}

Notes. One average radio flare is centered on each X-ray state with hardness $>0.001$ following a state with hardness $<0.001$ within 10 days since those are candidate radio flare events.

a $S_{15 \mathrm{GHz}}=$ measured radio flux density at $15 \mathrm{GHz}$.

${ }^{\mathrm{b}} S_{\mathrm{fit}}=\kappa \exp -\left(t-t_{0}\right)^{2} / 2 \sigma^{2}$, average of Gaussians fitted to 28 radio flares, $\kappa=4.81 \mathrm{Jy}, \sigma=1.16$ days.

a radio and an X-ray part, as in Table 1 . For the reconstruction of radio flares from X-ray data, an average radio flare was obtained from Gaussians fitted to 28 flares seen to rise above $1 \mathrm{Jy}$ in radio data. One average radio flare was put at each time of potential radio flaring, as seen in X-ray data (i.e., spectral hardness $>0.001$ within 10 days after a state with hardness < 0.001 ) to obtain a pseudo radio flux density $S_{\text {fit }}$. The resulting search windows from all selections can be overlapping and are combined with a logical OR operation, resulting in the final time windows. The utilized radio data were taken with the Arcminute Microkelvin Imager (AMI) radio telescope (Zwart et al. 2008; Pooley \& Fender 1997) at a frequency of $15 \mathrm{GHz}$ between 2008 May and 2009 May at irregular intervals. X-ray data were obtained from the Rossi X-ray Timing Explorer/All Sky Monitor (RXTE/ASM; Levine et al. 1996), using the $B$ band between 3 and $5 \mathrm{keV}$, and from the Burst Alert Telescope on board the Swift satellite (Swift/BAT) (Barthelmy et al. 2005) sensitive between 15 and $50 \mathrm{keV}^{47}$ The ratio of BAT (hard X-ray) to ASM B (soft $\mathrm{X}$-ray) counts provides the spectral hardness parameter. Gamma-ray data from Fermi or AGILE are not explicitly taken

\footnotetext{
47 AMI data from G. Pooley, http://www.mrao.cam.ac.uk/ guy/cx3/data/ (2010 January 14). RXTE/ASM data from H. Bradt, D. Chakrabarty, W. Cui et al., http://xte.mit.edu/ASM_lc.html (2010 March 11). Swift/BAT data from H. Krimm, http://swift.gsfc.nasa.gov/docs/swift/results/transients/CygX-3/ (2010 June 4).
}

Table 2

Search Windows Extracted from Radio and X-Ray Data for the Neutrino Search from Cyg X-3 Direction

\begin{tabular}{lccc}
\hline \hline Window & $\begin{array}{c}\text { Start } \\
(\mathrm{MJD})\end{array}$ & $\begin{array}{c}\text { Stop } \\
\text { (MJD) }\end{array}$ & $\begin{array}{c}\text { Duration } \\
\text { (days) }\end{array}$ \\
\hline 1 & 54571.4 & 54582.5 & 11.1 \\
2 & 54584.5 & 54607.4 & 22.9 \\
3 & 54637.6 & 54649.5 & 11.9 \\
4 & 54811.5 & 54824.6 & 13.1 \\
\hline
\end{tabular}

into consideration in this analysis. Applying the selection criteria described in Table 1 to the radio and X-ray data from the time when the 40 string IceCube was operating (between MJD 54560 and MJD 54989) results in the four time windows indicated in Table 2 and Figure 3. Even though there are no AMI data from the first 2.5 months of this period, an ATel (Trushkin et al. 2008) was issued for a strong radio flare around MJD 54574 that is consistent with the time windows selected from X-ray data.

The data have been analyzed with a maximum likelihood test using a time-dependent version of the unbinned likelihood ratio method (Braun et al. 2010). The search time windows are incorporated into the signal probability density function (PDF) of the likelihood function as normalized Gaussians with mean located at the window center and FWHM equal to the 
window duration. During the maximization, the windows were allowed to be shifted up to 20 days to earlier or later times. This allows us to find neutrino emission that comes before or after a radio flare. The value of 20 days is motivated by the hypothesis of emission during the radio quenched state, which can happen up to $\sim 20$ days before the onset of a major radio flare (Koljonen et al. 2010; Trushkin et al. 2007). As in the other analyses presented in this work, no energy estimator is used in the likelihood. In the search for neutrinos from the microquasar Cygnus X-3, five searches are performed in total: one with each of the four windows as a hypothesis of a neutrino signal light curve, shifting each window individually, and one search using all four windows, shifting the windows simultaneously. With this analysis, only about $50 \%$ of the discovery flux of a time-integrated search (that uses no information about the activity of Cyg X-3) is needed for a $5 \sigma$ discovery, assuming the neutrino emission happens during the windows or within \pm 20 days. The discovery flux with $50 \%$ detection probability is $d N / d E \approx 10^{-10} \mathrm{TeV}^{-1} \mathrm{~cm}^{-2} \mathrm{~s}^{-1}$ for an $E^{-3}$ spectrum and $d N / d E \approx 1.2 \times 10^{-11} \mathrm{TeV}^{-1} \mathrm{~cm}^{-2} \mathrm{~s}^{-1}$ for an $E^{-2}$ spectrum.

\section{NEUTRINO SAMPLES}

Here we discuss the selection of the sample of neutrino candidates for each of the two detector configurations considered. While the event selection is optimized for each of these two configurations separately, both analyses are based on the selection of events on the basis of track reconstructions. We therefore first discuss the concepts that are common to the two event selections: the track reconstruction and its application to onand offline event selection as well as a special filtering of the AMANDA data.

\subsection{Track Reconstruction and Event Filtering}

At energies above $\approx 200 \mathrm{GeV}$, the identification of neutrino candidate events in the IceCube and AMANDA data is based on the selection of well-reconstructed up-going events since the dominant background is muons from cosmic-ray air showers, which reach the detector in the downward direction but are filtered out by the Earth in the upward direction, leaving only neutrinos.

The IceCube and AMANDA data are divided into two streams that are treated in a similar way. The first stream consists of events that trigger AMANDA. As explained above, these events are then complemented with the data collected in IceCube and for this reason they are considered combined events (C-events). The second stream consists of those events that trigger only IceCube (ICO-events), either because the number of hits in AMANDA is below the trigger threshold or because AMANDA was not active at the time the event was recorded.

Initial fast track reconstructions and event selections are applied online at the South Pole since the available bandwidth for satellite transfer of data is limited. For ICO-events, a straight track is fit to the data by minimizing the distance between the track and the hits (linefit; Ahrens et al. 2004). For C-events, a pattern recognition algorithm (called JAMS) has been used (Ackermann 2006). The online filters are based on these reconstructions and the relevant events for the presented analysis are selected by requiring that the first-guess reconstruction is not down-going. At this level, the data are still dominated by atmospheric muons that are mis-reconstructed as ascending. In particular, coincidences between multiple muons from different cosmic-ray air showers can mimic ascending event topologies.
After transfer to the north, more sophisticated reconstructions are applied to improve the angular resolution and to provide quality parameters for the rejection of the down-going atmospheric muon background. These reconstructions are maximum likelihood fits that are based on the probability density function for the arrival time of a photon given the track hypothesis (van Eijndhoven et al. 2007). Two likelihood reconstructions have been studied: one based only on the first photon in each optical module (single photoelectron, SPE) and a more complete one that includes the possible presence of multiple photoelectrons (MPE). For more details about the SPE and MPE reconstructions, we refer to Ahrens et al. (2004). A list of variables that are indicative of the quality of the reconstructed event is given in Abbasi et al. (2011a). In addition to the track reconstruction itself, an estimate of the angular uncertainty of the track reconstruction is obtained for each individual event by the evaluation of the likelihood function near the maximum. This method is described in Neunhöffer (2006). The estimate of the angular uncertainty is used for the event selection as well as in the maximum likelihood ratio test.

In the IC40+A analysis, we also make use of an energy reconstruction that is based on the characterization of the energy loss along the particle track. At energies above a few hundred $\mathrm{GeV}$, the energy loss of a muon in the ice is proportional to its energy. The energy reconstruction used in this work is presented in Zornoza \& Chirkin (2008).

\subsection{AMANDA Data}

In contrast to IceCube, the waveforms collected with the optical modules of AMANDA are not digitized directly in the optical modules but are transferred to the surface as analog signals. This introduces two undesired effects in the data. The first one is crosstalk between different cables. Large pulses in one optical module can cause a detectable signal within the electronics of cables connected to other optical modules.

The second issue concerns the pickup of electromagnetic noise. The PMT signals have to be transferred over a distance between $1.5 \mathrm{~km}$ and $2.0 \mathrm{~km}$ to the surface. The cables needed for this task are vulnerable to picking up electromagnetic noise as they act as electromagnetic antennae.

Methods dedicated to the identification of non-particleinduced signals based on the waveforms have been developed and result in an efficient separation from particle-induced signals. The integral over the entire collected waveform pulse is used in order to remove crosstalk pulses. Since crosstalk pulses do not originate from a charge deposit in the PMT, they consist of (positive and negative) fluctuations around the baseline with the total integral close to zero. Waveforms from a particle-induced signal in the PMT have a characteristic width, which is wider at the surface due to dispersion in the cables. For AMANDA strings 5-10, this is typically 250-300 ns for SPEs. Waveforms from MPEs result from the (linear) overlay of many SPE waveforms and are typically wider than SPEs. In contrast, noiseinduced waveforms are often very spiky, i.e., they have many peaks within the width corresponding to a typical SPE pulse. This feature of non-particle-induced waveforms has been used to remove noisy events from the data. Events that simultaneously have a median peak rate in the waveforms recorded in AMANDA strings 5-10 which is incompatible with a PMT signal (above $5 \mathrm{MHz}$ ) and a high number (more than 20) of waveforms in these strings, are considered non-particle-induced and are removed from the data set. Figure 4 illustrates this cut. Both analyses presented in this paper first apply cross-talk 
run 00110594

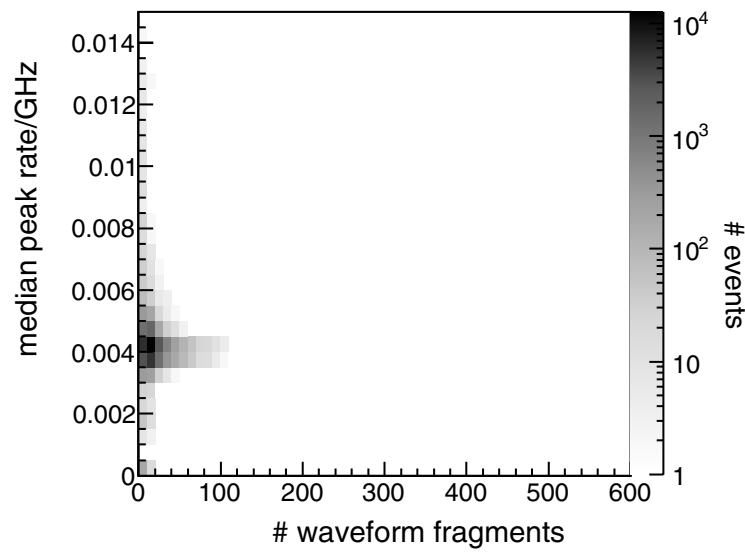

run 00109325

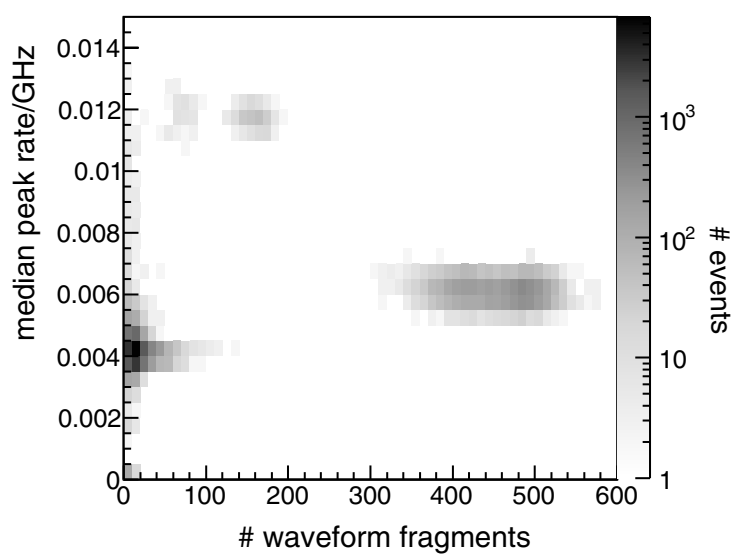

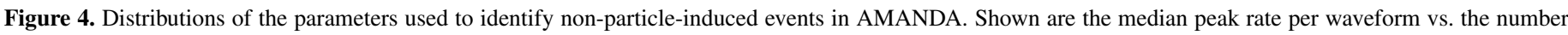

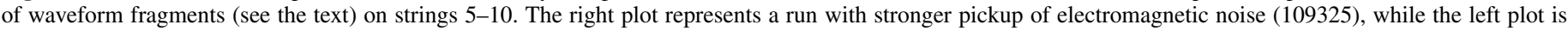

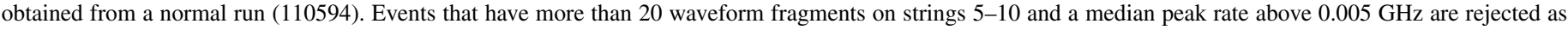
non-particle-induced events.

cleaning and the above technique for rejection of non-particleinduced events to the AMANDA data, before further event selections are made.

\subsection{String Ice Cube and AMANDA}

Data have been collected from 2007 May 31 to 2008 April 4 when IceCube was operating in a 22 string configuration. The lifetime of the IC22+A run is 276 days, including 143 days of AMANDA operating in stable mode. The unusually long downtime of AMANDA during this period was caused by various hardware failures during 2007 May (trigger system) and during 2007 August (high voltage supply system). In this section, the event selection from the trigger level up to the final analysis level is described and the characteristics of the combined neutrino sample are highlighted.

\subsubsection{Trigger and Online Filter}

As explained above, IceCube and AMANDA are triggered separately in the combined detector mode. The trigger rate of IC22 is $550 \mathrm{~Hz}$, while AMANDA triggers at $200 \mathrm{~Hz}$. Seasonal variations affect the trigger rate by about $10 \%$. The overall trigger rate of the combined $\mathrm{IC} 22+\mathrm{A}$ detector after correction for overlaps between the two triggers is $640 \mathrm{~Hz}$. At trigger level, the data are strongly dominated by down-going atmospheric muons induced by cosmic-ray air showers outnumbering atmospheric neutrinos by a factor of about $10^{6}$. The online filter reduces the data volume for satellite transfer and has a passing rate for reconstructed up-going or horizontal events of $22 \mathrm{~Hz}$ for ICOevents and $8 \mathrm{~Hz}$ for $\mathrm{C}$-events, producing a total event rate of $30 \mathrm{~Hz}$.

\subsubsection{Neutrino Sample}

After the rejection of down-going reconstructed events based on first guess reconstructions, the data are still dominated by mis-reconstructed atmospheric muons and further event selections are needed in order to arrive at a sample of events dominated by atmospheric neutrinos. A typical quality parameter used in this analysis is the number of un-scattered photons, socalled "direct hits," which are characterized by a small time residual with respect to the expectation of the geometry of the emitted Cherenkov cone.
In order to optimize the retention of lower energy events we have employed a multivariate approach. In this approach, a signal likelihood is defined as the product of the signal likelihood from the variables considered and is compared to the likelihood of the background hypothesis. According to the Neyman-Pearson lemma (Neyman \& Pearson 1933), this criterion leads to the best possible discrimination power for the given set of variables if there are no correlations between them. For correlated variables, as in our case, this criterion still turns out to be powerful. We use experimental data dominated by atmospheric muons to describe the background, and simulated neutrinos weighted to an atmospheric neutrino spectrum in order to model the signal. C-events and ICO-events are treated independently here. For ICO-events, further cuts are used to reject coincident air shower muons. These cuts are based on the smoothness of the distribution of the hits along the track and on reconstructions performed on subsets of hits. For C-events, the rate of coincident muons is significantly lower due to the smaller size of AMANDA. While a tighter time-window cleaning helped to further reject the coincident muons in the combined events stream, no dedicated cuts/reconstructions have been used to remove these.

The final cuts were optimized for the best discovery potential and finally tracks are selected if the angular resolution estimator returned a value lower than $4^{\circ}$. A harder cut on this parameter did not lead to an improved discovery potential.

The resulting neutrino sample contains about 1.8 times more events than the search presented in Abbasi et al. (2009a). In total, 8727 events are selected, of which 3430 are C-events. These event numbers correspond to a data rate of $4.7 \times 10^{-4} \mathrm{~Hz}$ for $\mathrm{IC} 22+\mathrm{A}$ and to $2.4 \times 10^{-4} \mathrm{~Hz}$ in IC22 only mode. The effective area of the IC22+A analysis is shown in Figure 5. Below energies of a few $\mathrm{TeV}$, there is a strong improvement when including AMANDA, with respect to the performance of a low-energy optimized analysis using IceCube only. The energy distribution of atmospheric neutrinos for this selection according to simulations is shown in Figure 6.

The estimated angular resolution is given by a median of 1.9 for atmospheric neutrinos and 1.7 for a Crab-like spectrum. About $10 \%$ of the final events are expected to be mis-reconstructed muon background.

The minimum detectable flux is more than an order of magnitude above the neutrino emission expected from the Crab 

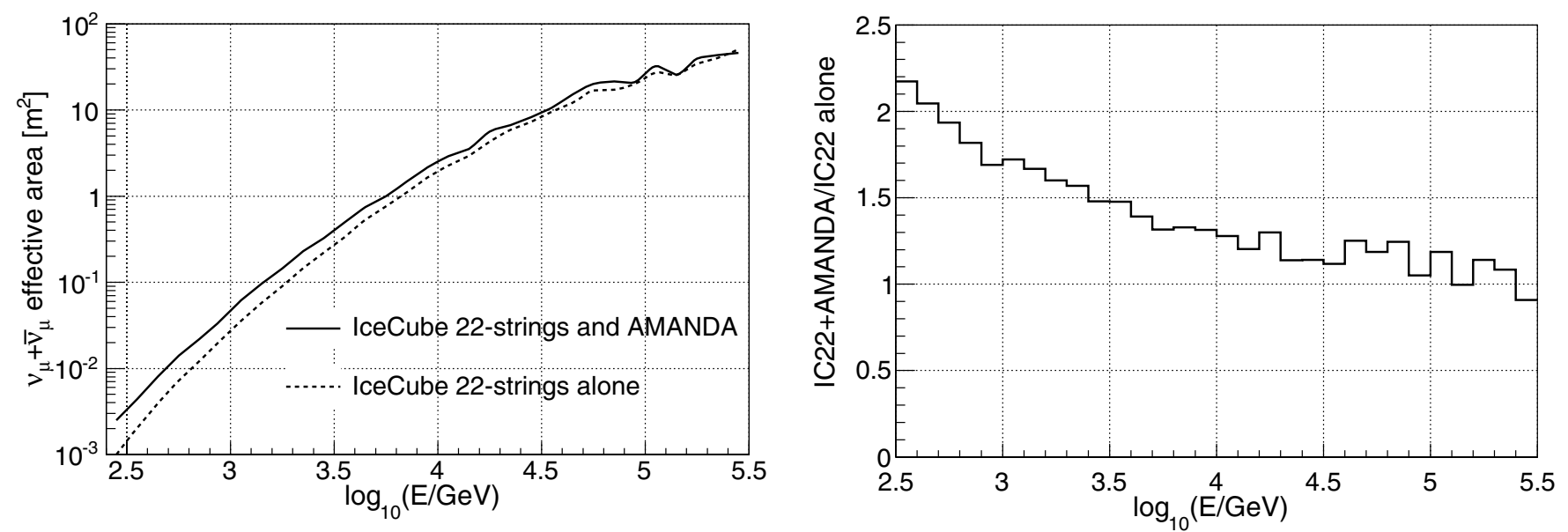

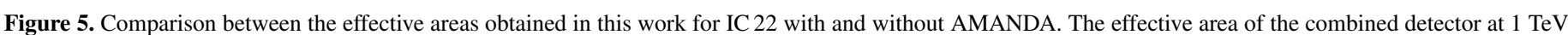
is a factor $\approx 1.6$ larger than without AMANDA. The last bins of the ratio plot are affected by limited statistics.

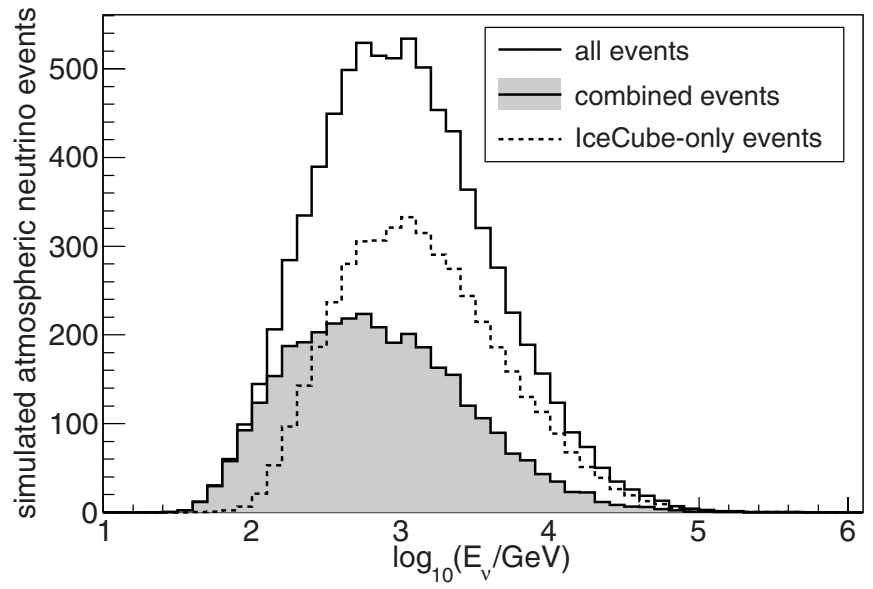

Figure 6. Energy distribution for simulated atmospheric neutrinos at the final selection level of the Galactic point-source analysis with IC22+A normalized to the lifetime of the IC22 data.

assuming that the H.E.S.S. observations are consistent with a model of $p p$ interactions. While with this expectation, a positive detection is unlikely but not excluded as the photon flux may be absorbed, the analysis is valuable as a starting point for future improvements with the full IceCube detector and the DeepCore sub-detector. Using an $E^{-3}$ spectrum for the comparison, the discovery flux in this analysis is between $15 \%$ and $33 \%$ lower than the one in Abbasi et al. (2009a), depending on the declination. This clearly demonstrates the improvement obtained by the combined use of a low-energy core and the optimization of the event selection for softer spectra. An additional improvement is obtained by the lower number of trials in the Galactic plane scan versus the scan of a hemisphere or the whole sky but is not quantified here.

\subsection{String IceCube and AMANDA}

Following the explorative analysis strategy developed for the IC22+A, a similar analysis has been conducted on the larger data sample collected with the combined IC40+A detector from 2008 April 5 to 2009 May 20. Both parts of the combined IceCube-AMANDA detector operated very stably during this time period. For IC40 about 375 days of data were collected and used in this analysis and for AMANDA about 306 days. The main causes for downtime were scheduled operations in the course of the integration of new strings into the detector. Moreover, the decommissioning of AMANDA began a few weeks before the completion of the IC40 run. The event selection is in many respects similar to the one applied to the IC22+A data as the targeted energy range is the same as well as the physics driving the analysis. Again, different cut criteria are developed for $\mathrm{C}$ - and ICO-events.

\subsubsection{Trigger and On-line Filter}

For this analysis, the trigger logic was kept identical to the one in the previous season. The online filter selected about $20 \mathrm{~Hz}$ of track-like ICO-events and $3 \mathrm{~Hz}$ of upgoing C-events.

\subsubsection{Neutrino Sample}

Similar to the previous combined analysis, ICO-events are selected by a series of one-dimensional cuts on event quality parameters and combined with a multivariate classification based on the Neyman-Pearson lemma (Neyman \& Pearson 1933). The probability density functions for five quality parameters are generated from down-going atmospheric muon-dominated data as background and from up-going atmospheric neutrino simulations as signal, and combined in the cut. (The fraction of atmospheric neutrino events in the data is still only about $4 \%$ and the data can therefore be regarded as dominated by background atmospheric muons.) The five quality parameters used in this analysis are: the quality parameter of the likelihood reconstruction, an estimate of the angular uncertainty of the likelihood reconstruction obtained by the evaluation of the likelihood space around the maximum, and three variables that describe the amount and distribution of un-scattered light in the event. A PMT pulse from un-scattered light is characterized by a small time residual with respect to the expectation from the geometry of the Cherenkov cone. The number of PMT pulses with time residuals between $-15 \mathrm{~ns}$ and $75 \mathrm{~ns}$, the maximum distance between their projections on the reconstructed track and the smoothness of their distribution along the track of the particle are used in the event selection. More information about these variables is reported in Abbasi et al. (2011a). The distribution of the resulting cut variable is shown in Figure 7 for data and for atmospheric neutrino simulation as well as for two example signal neutrino spectra. An optimization of the discovery potential for a soft $E^{-3}$ spectrum results in an optimal cut value of 1.0 . 


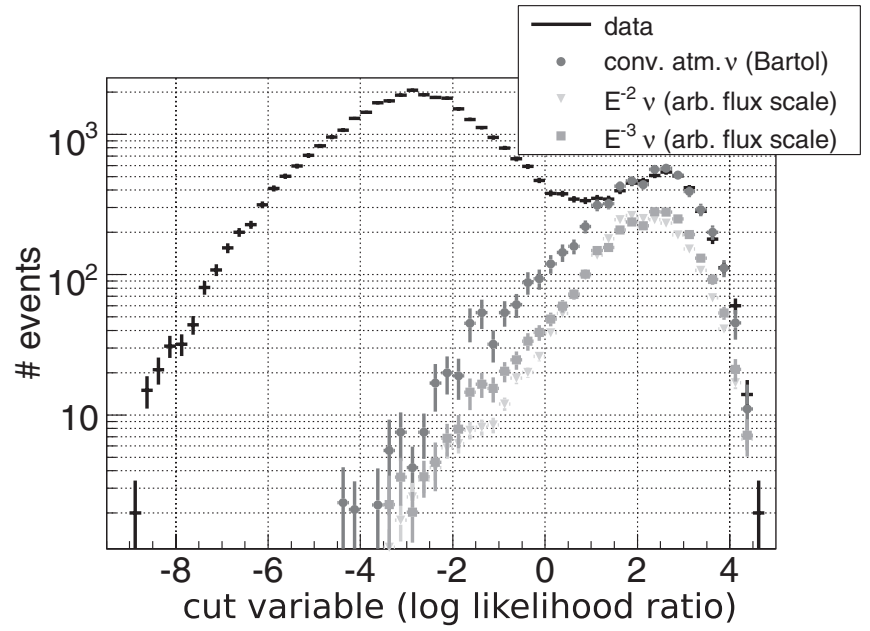

Figure 7. Distribution of the main cut variable for IceCube events in the 40 string IceCube and AMANDA analysis. Data are shown along with simulated atmospheric neutrinos (Barr 2004) and two different simulated neutrino signals of arbitrary flux scale. No normalization is applied. A cut at a value of 1.0 is applied to the data to select a neutrino sample.

C-events are first cleaned as described in Section 4.2. Subsequently, a series of one-dimensional cuts is applied. This series of cuts has been challenged versus other more sophisticated cut strategies and demonstrated to perform well enough in the separation of the atmospheric muon background and the neutrino signal.

While the analysis is optimized for soft spectra such as $E^{-3}$ or the Crab-spectrum, it is nevertheless desirable to retain a good efficiency for very high energy neutrino events as well. With a cut optimized on a very soft spectrum, however, the retention of high-energy events is not necessarily optimal as these deposit much more light in the detector and may thus have event topologies that are not caught in the low-energy event selection. To remedy this, additional criteria are included in the event selection if the signature is likely to be induced by a very high energy neutrino. These additional events are selected in both streams with a series of one-dimensional cuts based among others on their reconstructed energy.

The IC40+A analysis also uses maximum likelihood track reconstructions. For low energetic combined IceCube-AMANDA events, an SPE PDF is used and for all other events, an MPE PDF is used.

The total number of selected neutrino candidates is 19,797 in the entire lifetime of 375 days. The purity of the atmospheric neutrino sample is estimated to be $97 \%-98 \%$. Of the selected neutrino candidates, $81.3 \%$ are ICO-events selected with the multivariate Neyman-Pearson likelihood ratio cut. $2.4 \%$ are additional ICO-events with high estimated energies. The remaining $16.3 \%$ events are C-events. Despite the larger lifetime of AMANDA in 2008/2009 with respect to the previous year, the fraction of C-events in this analysis is smaller than in the previous one. This is partially due to the larger size of the IceCube detector but also to the higher purity of the IC40+A sample. The resulting energy distribution as derived from atmospheric neutrino simulation is shown in Figure 8. The selected C-events peak at lower energies than the ICO-events. Also the effective area, reported in Figure 9, shows the effect of AMANDA at lower energies. With the larger size of the detector, the effective area is improved significantly with respect to the IC22 analysis.

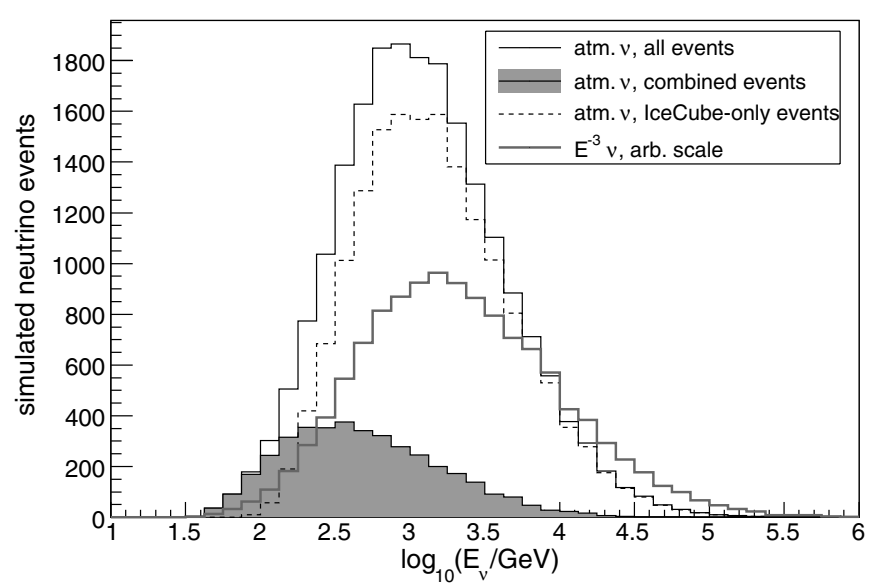

Figure 8. Energy distribution of the final neutrino sample obtained in the 40 string IceCube and AMANDA data sample. This comparison is based on simulated neutrinos following the Bartol model (Barr 2004).

Figure 10 shows the distribution of the declinations of the events in the final neutrino sample. The angular resolution obtained with this selection of events is shown in Figure 11, together with the angular resolution of the IC22+A analysis. The sensitivity and discovery potential for $E^{-3}$ neutrino spectra are reported in Figure 12.

\section{RESULTS}

As a result of the integrated use of the AMANDA detector within IceCube, we have obtained a significant improvement in the retention of neutrino-induced events below a few $\mathrm{TeV}$ (see Figure 9). This region is of importance for sources with soft or cutoff spectra. The tests described above resulted in no evidence for significant deviation from the background-only hypothesis. In the absence of detection of an astrophysical neutrino signal, upper limits on the muon neutrino flux from the considered regions of the Galaxy have been determined. All the upper limits have been derived for soft neutrino spectra, with and without energy cutoff. The $90 \%$ confidence level limits $\left(\Phi_{v_{\mu}}^{90 \%}\right)$ are calculated using the method of Feldman \& Cousins (1998), i.e., $d \Phi_{v_{\mu}} / d E \leqslant \Phi_{v_{\mu}}^{90 \%}(E / \mathrm{TeV})^{-\alpha} \mathrm{TeV}^{-1} \mathrm{~cm}^{-2} \mathrm{~s}^{-1}$. Systematic uncertainties have been included in the limit determination using the method defined in Conrad et al. (2003) with the modification in Hill (2003).

The upper limits are calculated for the total of the muon neutrino and antineutrino flux reaching Earth, assuming that no other neutrino flavors contribute to the possible signal. For a source producing muon and electron neutrinos in the ratio of about $v_{e}: v_{\mu}: v_{\tau}=1: 2: 0$ typical of pion production from $p p$ or $p$-gamma interaction, neutrino oscillations with a large mixing angle $\theta_{23} \sim 45^{\circ}$ and long baseline result in approximate equipartition of flavors. This analysis is to some extent sensitive to $v_{\tau}$ as well, mainly due to the decay of a $\tau$ into a $\mu$ with a branching ratio of $\approx 17 \%$. Taking into account these effects and the details of energy losses, the contribution of $v_{\tau}$ is estimated to be an additional $10 \%-16 \%$ of the $v_{\mu}$ contribution for IceCube (Abbasi et al. 2011d) and AMANDA analyses (Abbasi et al. 2009c).

After completion of this analysis, a slight overprediction of the muon neutrino flux has been observed by comparing the results with an improved Monte Carlo simulation not previously available. The intensity of the effect varies in declination and energy and it is estimated to be less than $30 \%$. As a consequence, 

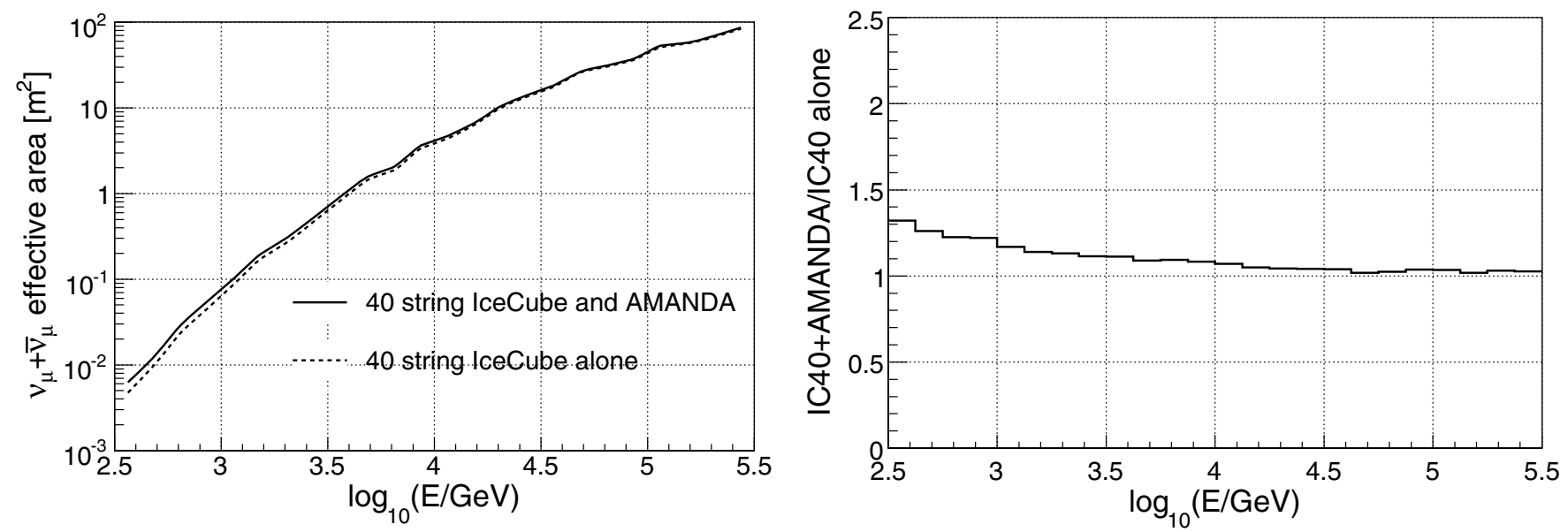

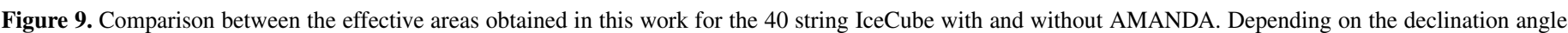

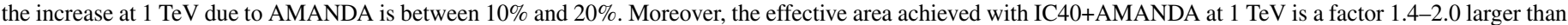
that in the IceCube-40-only analysis (Abbasi et al. 2011a), due both to the inclusion of AMANDA and to an event selection optimized for lower energies.

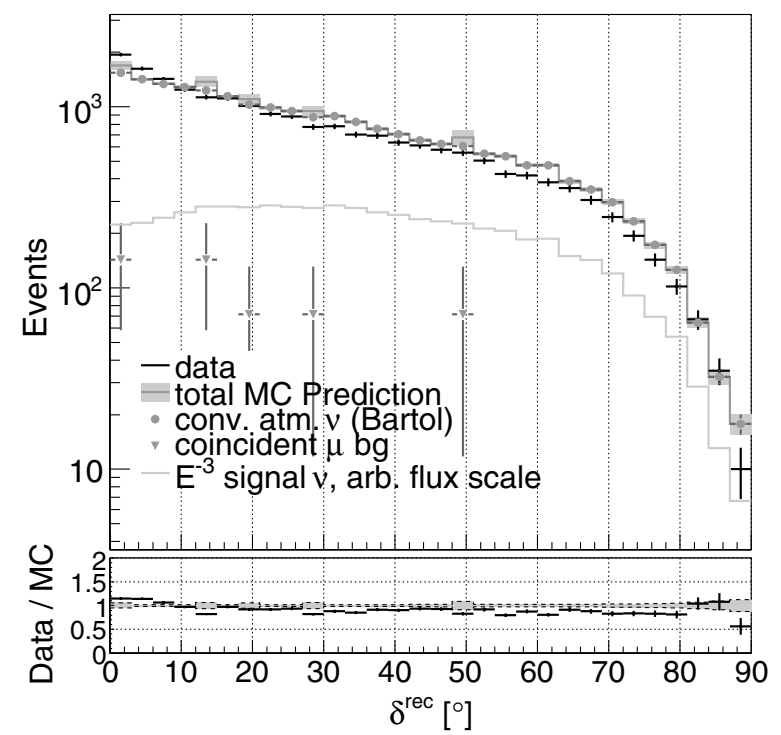

Figure 10. Comparison of experimental data with Monte Carlo simulation for the 40 string IceCube and AMANDA analysis. All error bands are purely statistical. After considering the systematic uncertainties described in the text, the declination distribution of the final neutrino sample is in agreement with the expectations of atmospheric neutrinos.

all upper limits reported in this work as well as in Abbasi et al. (2011a, 2011d) are slightly overconstraining.

\subsection{Galactic Plane Scan and Steady Sources}

In Figures 13 and 14, we present the results of the scan of the Galactic plane with IC22+A and with IC40+A. We note here that the region of the Galactic plane considered covers only around $1 / 23$ of the whole sky. This restriction in the tested area results in a lower trial factor compared to a $4 \pi$ map. Under the conservative assumption of a uniform angular resolution, the effective number of trials is expected to be a factor of 23 lower than in an All-Sky scan like the one realized in Abbasi et al. (2011a). Assuming that the relation between the post-trial $p$-value $p_{\text {post }}$ and the pre-trial $p$-value $p_{\text {pre }}$ is $p_{\text {post }}=1-\left(1-p_{\text {pre }}\right)^{N_{\text {eff }}}$, the effective number of trials in the IC40+A search is about $N_{\text {eff }} \approx 2200$. The reported posttrial $p$-values have been obtained by performing the analysis on randomized, and therefore signal-free, data samples.

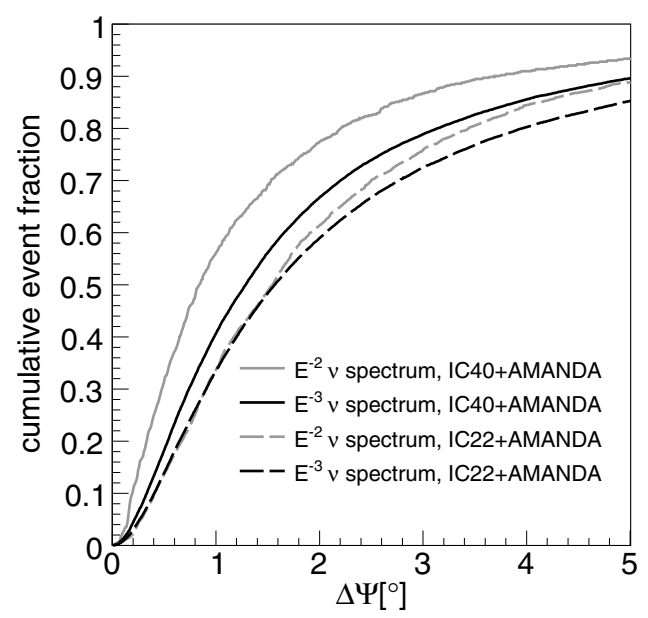

Figure 11. Angular resolution of the two configurations, IceCube-22 and IceCube-40, combined with AMANDA. The angular resolution is determined for two different possible signal spectra. The $E^{-2}$ neutrino spectrum includes on average events with higher neutrino energies and better angular reconstruction. With the IC40+AMANDA configuration, $90 \%$ of the neutrino events with an $E^{-2}$ neutrino spectrum are in the energy interval from $2.4 \mathrm{TeV}$ to $750.0 \mathrm{TeV}$ while the energy region from $0.2 \mathrm{TeV}$ to $20.5 \mathrm{TeV}$ contains $90 \%$ of the neutrino events if an $E^{-3}$ spectrum is considered.

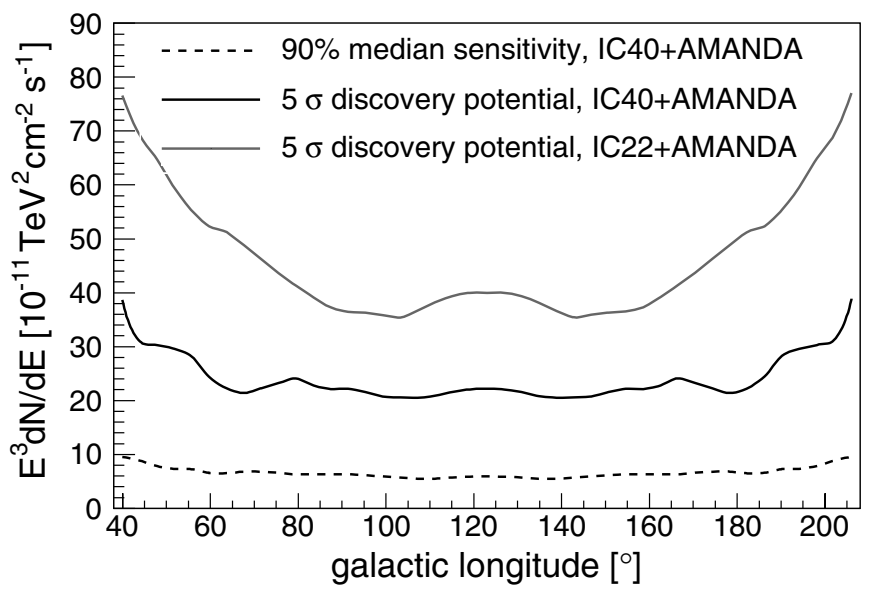

Figure 12. Sensitivity and discovery potential of the IC40+AMANDA analysis for $E^{-3}$ neutrino spectra. The sensitivity is calculated with the method of Feldman \& Cousins (1998) with a systematic uncertainty of $\pm 17 \%$ on the neutrino flux included using the method defined in Conrad et al. (2003) and modified in Hill (2003). 


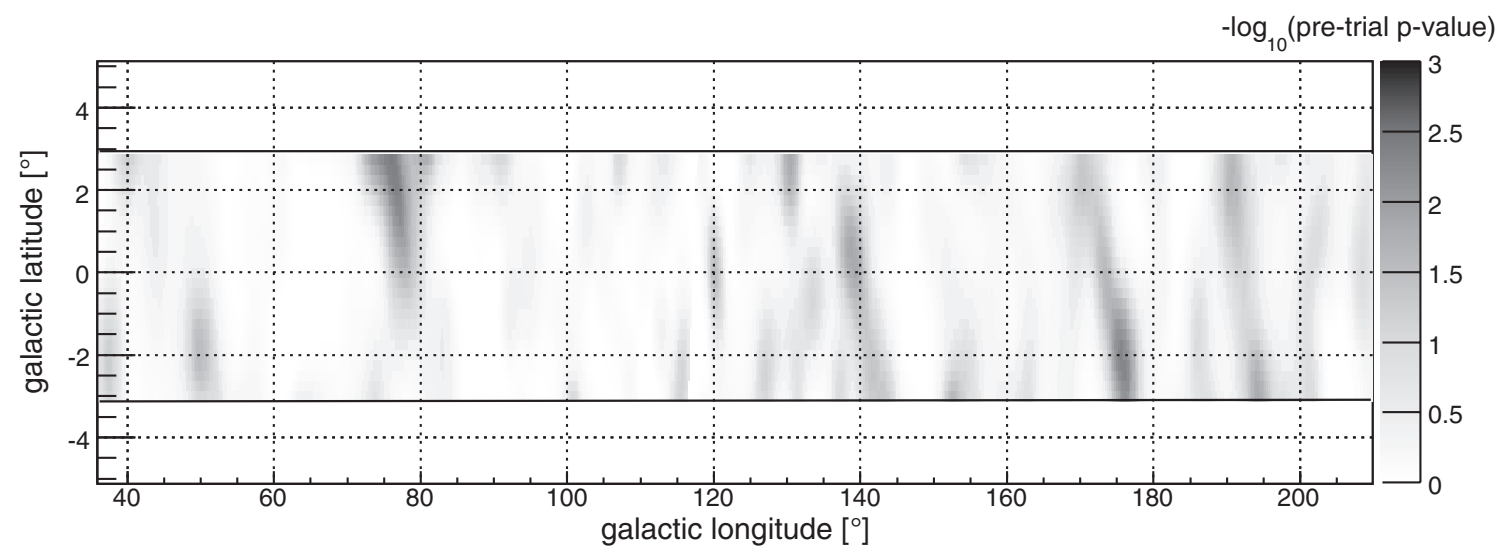

Figure 13. Pre-trial significances ( $p$-value) of the Galactic plane scan using the 22 string IceCube and AMANDA data.
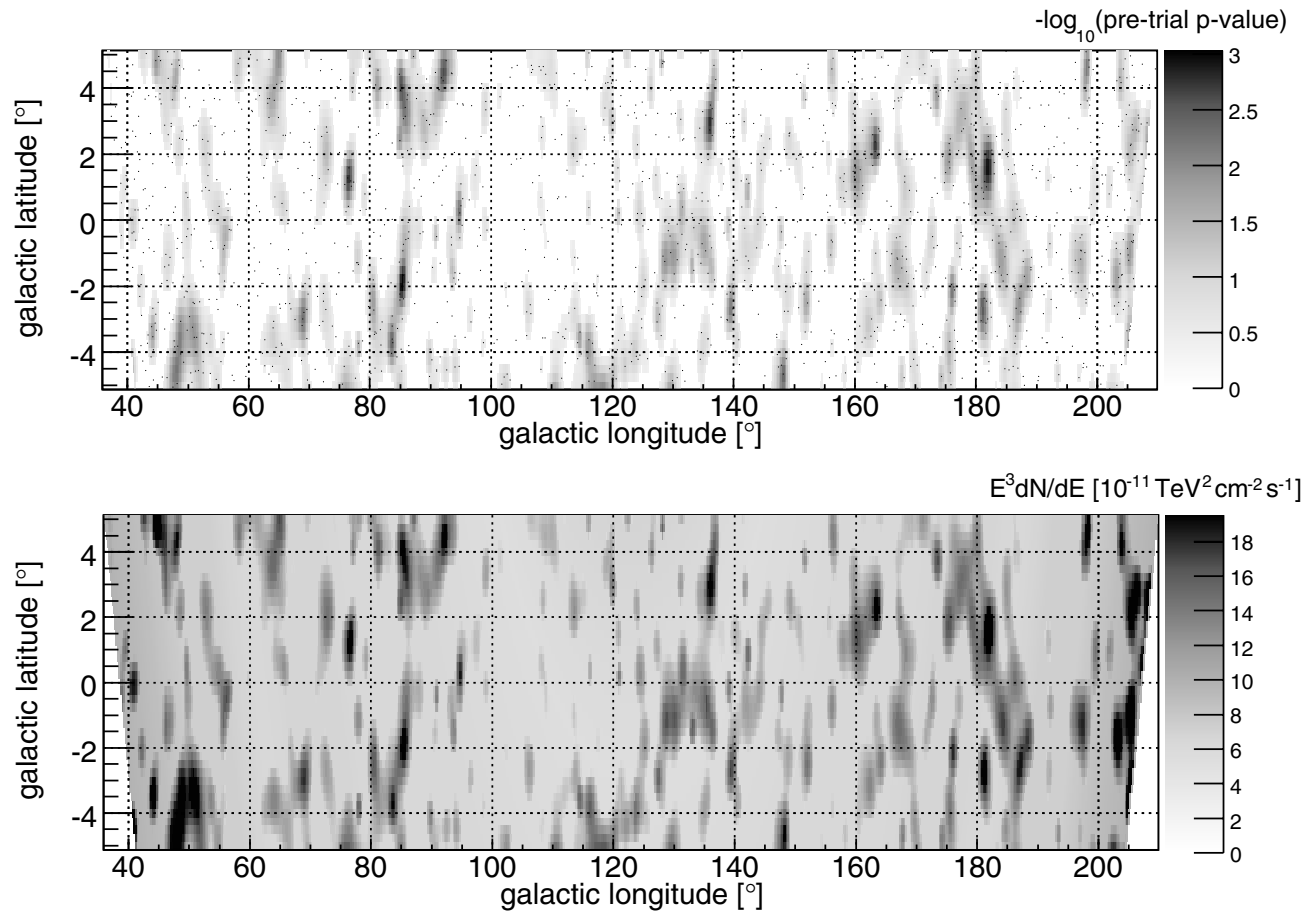

Figure 14. Result of the scan of the Galactic plane with data collected by IceCube- 40 combined with AMANDA. Above: At each $0.25 \times 0.25$ grid point, the result of the maximum likelihood analysis is shown in terms of its estimated significance $\left(-\log _{10}\right.$ pre-trial $p$-value). The reconstructed event locations are also indicated as black dots. The most significant excess of events is located at Galactic coordinates $\left(85.5,-2,0^{\circ}\right)$ and has a pre-trial $p$-value of $0.09 \%(-\log (p$-value $)=3.03)$. Accounting for the trials induced by the scanning of many points inside the Galactic plane, the $p$-value of this test is $88 \%$. Below: upper limits map for a signal spectrum proportional to $E^{-3}$.

In the analysis of IC22+A, the lowest background probability at point-source angular scales is found at Galactic coordinates $\ell=75.9, b=2.7$, with a pre-trial $p$-value of $0.37 \%$. Considering the intrinsic trials of the scan by analyzing randomized data samples, an equal or higher significance in at least one of the scanned locations is found in $95 \%$ of the cases. The most significant point-like spot in the analysis of IC40+A, with a pre-trial $p$-value of $0.09 \%$, is found at Galactic coordinates $\ell=85.5, b=-2.0$. An equal or higher significance is found in $88 \%$ of the randomized data samples.

Upper limits on the neutrino emission have been calculated for the six pre-selected candidate neutrino sources that have been studied for steady neutrino emission and are summarized in Table 3 for an $E^{-3}$ spectrum without cutoff. These limits have been obtained from the IC40+A analysis.
Table 3

$E^{-3}$ Muon Neutrino Flux Upper Limits ${ }^{\text {a }}$ from Six $\gamma$-ray Sources, Based on 40 String IceCube and AMANDA

\begin{tabular}{lrrccc}
\hline \hline Object & \multicolumn{1}{c}{ R.A. } & Decl. & $n_{s}$ & Pre-trial $p$-value & $\Phi_{v_{\mu}}^{90 \%}$ \\
\hline Crab Nebula & 83.63 & 22.02 & 0 & - & 7.3 \\
LSI +61 303 & 40.13 & 61.23 & 1.6 & 0.25 & 8.3 \\
W51 & 290.82 & 14.15 & 0.6 & - & 8.3 \\
CasA & 350.85 & 58.82 & 0 & - & 5.9 \\
SS433 & 287.96 & 4.98 & 0 & - & 9.8 \\
IC443 & 94.18 & 22.53 & 0 & - & 7.3 \\
\hline
\end{tabular}

Note. ${ }^{\text {a }}$ The flux limits are given as $\Phi_{v_{\mu}}^{90 \%}$ in units of $10^{-11} \mathrm{TeV}^{-1} \mathrm{~cm}^{-2} \mathrm{~s}^{-1}$ and represent the $90 \%$ C.L. upper limit on the differential muon neutrino flux such that $d \Phi_{v_{\mu}} / d E \leqslant \Phi_{v_{\mu}}^{90 \%}(E / \mathrm{TeV})^{-3} \cdot p$-Values above 0.5 are given as "-." 

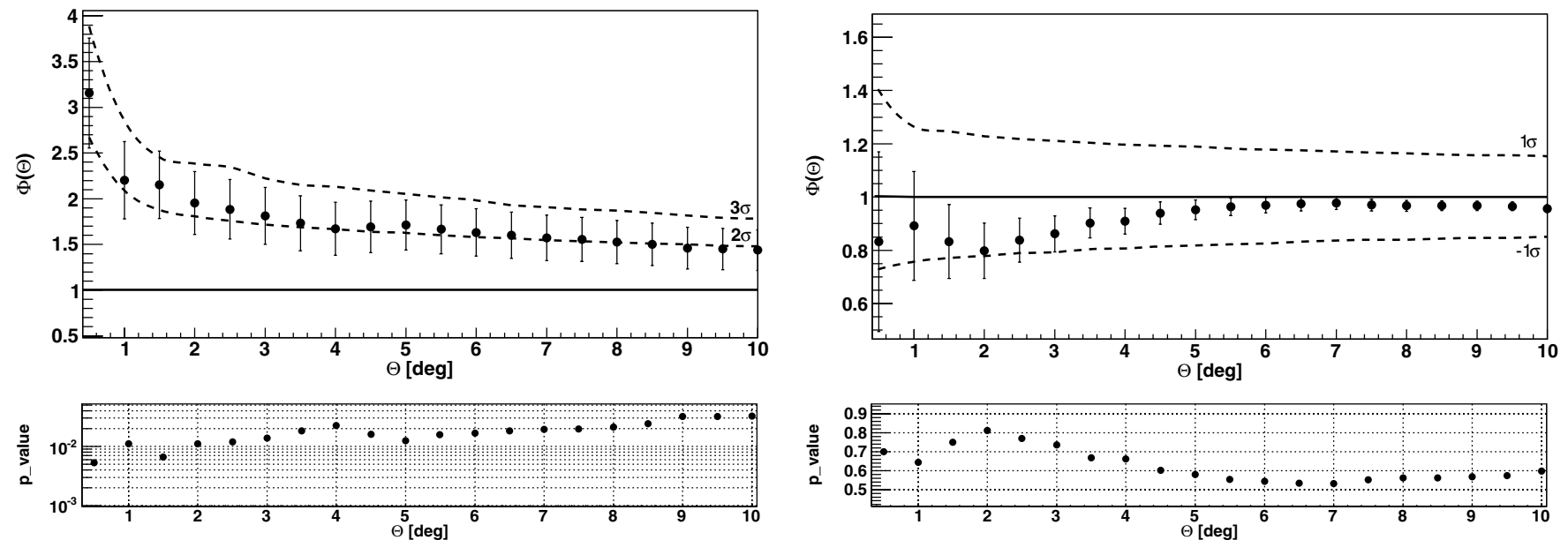

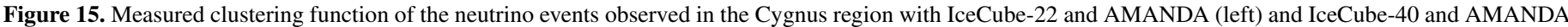

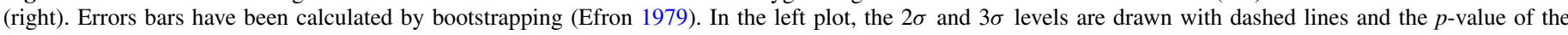
clustering function at each of the angular scales tested is also shown. For IceCube-40 on the right, the $1 \sigma$ level is indicated by the dashed line.
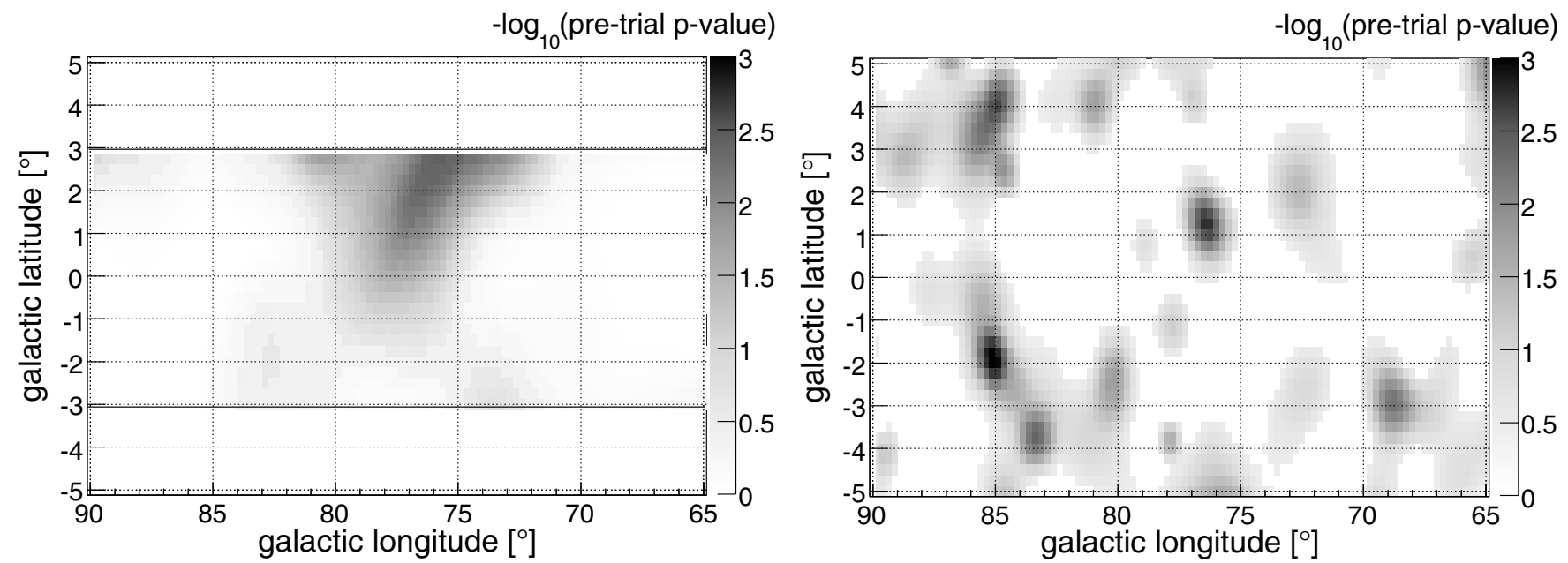

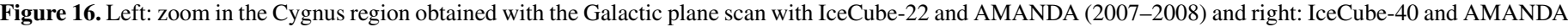
(2008-2009). The gray shading indicates the negative logarithm of the $p$-value before trial correction.

In $\mathrm{IC} 22+\mathrm{A}$, the highest excess in the candidate list was observed at the position of the Crab Nebula, with a pretrial $p$-value of $13 \%$ (37\% post-trial). In IC40+A, the most significant clustering of events was observed for LSI +61 303. The estimated number (best-fit value) of signal events $n_{s}$ from this location is 1.6 and the observation corresponds to a pretrial $p$-value of $25 \%$. Accounting for the trials from testing six different positions, the post-trial $p$-value of this search is $42 \%$, i.e., $42 \%$ of randomized data samples show a similar or stronger accumulation of events around one of the six objects.

\subsection{The Cygnus Region}

The clustering function $\Phi(\Theta)$ described in Section 3.2 has been computed for events with Galactic coordinates within $72^{\circ}<\ell<83^{\circ}$ and $-3^{\circ}<b<4^{\circ}$ for both IC22+A and IC40+A data. Figure 15 shows the clustering function of the events in each sample, that is, the ratio of the number of event pairs separated by angular distance $\Theta$ or less, with respect to the average number of such pairs for randomized events (i.e., the average case would therefore be located at $\Phi(\Theta)=1$ ). In the figure, we show the $2 \sigma-3 \sigma$ (IC22+A sample) and $\pm 1 \sigma$ $(\mathrm{IC} 40+\mathrm{A})$ levels of the distribution of $\Phi(\Theta)$ under the hypothesis of a random distribution of events. The observed values of $\Phi(\Theta)$ are represented together with measurement errors obtained by bootstrapping (Efron 1979). No significant concentration of events is seen at any of the angular scales tested. The result obtained on the IC22+A data sample shows a positive fluctuation at the level of $2.3 \sigma$. This result already contains the correction of the trials obtained via scrambling and associated with the observation at different angular scales. The region considered showed an excess with respect to the background expectation, which translates into excess values of $\Phi(\Theta)$ at all angular scales, but no significant structure is observed. The image of the Cygnus region obtained in IC22+A is shown in Figure 16 (left). However, the IC40+A analysis yields an underfluctuation within the analyzed area, showing a rather dispersed distribution of events with respect to the average background case and its image is in Figure 16 (right).

The conclusions from both the Galactic plane scans and the correlation analysis are therefore that the variations in the event density in the $11^{\circ} \times 7^{\circ}$ region analyzed are consistent with background fluctuations.

The underfluctuation observed in $\mathrm{IC} 40+\mathrm{A}$ provides restrictive upper limits to point-like neutrino emission above $500 \mathrm{GeV}$ from the Cygnus region. Upper limits have been computed from the measured value of the clustering function at 

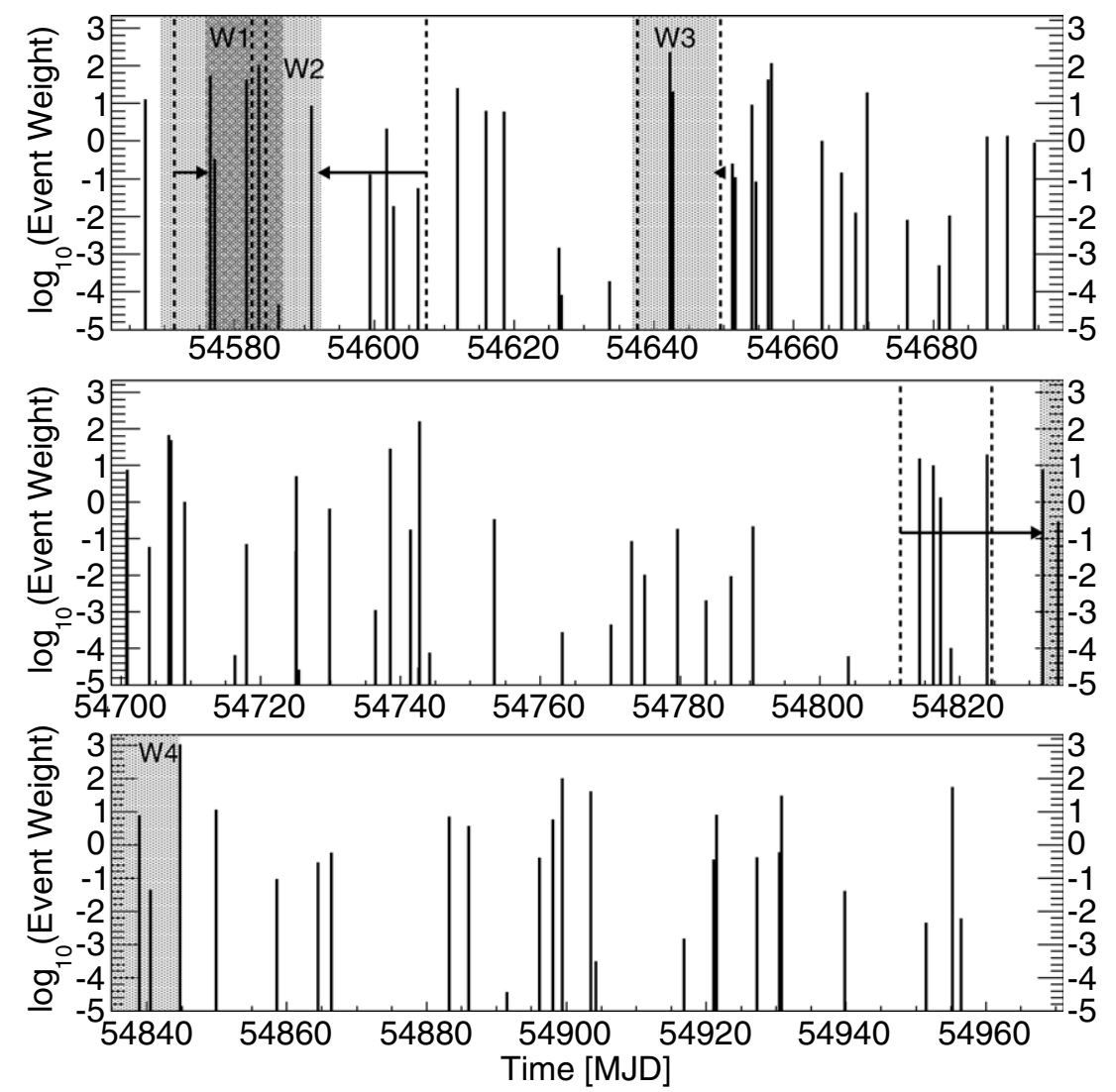

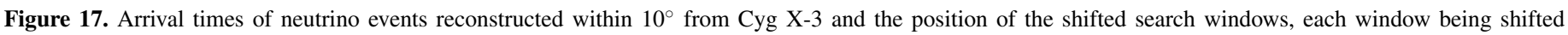

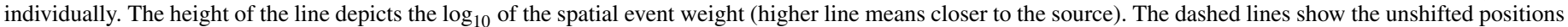
of the windows. The arrows indicate the shift.

Table 4

Feldman-Cousins Upper Limits ${ }^{\mathrm{a}}$ on Neutrino Flux from Cyg X-3, Averaged over the Whole Period of Data Taking, Assuming Emission Occurred Only during the Search Windows (i.e., Fluence Divided by Data-taking Time)

\begin{tabular}{lrrccccc}
\hline \hline Search & Shift & Events & Exp. & $n_{s}$ & Pre-trial & \multicolumn{2}{c}{$\Phi_{v_{\mu}}^{90 \%}$} \\
\cline { 5 - 8 } Window & (days) & & Background Events & & $p$-value & $E^{-3}$ & $E^{-2}$ \\
\hline 1 & +4.46 & 5 & $2.6 \pm 1.3$ & 1.6 & 0.22 & 4.7 & 0.73 \\
2 & -15.05 & 5 & $4.6 \pm 1.9$ & 0.8 & 0.42 & 4.0 & 0.60 \\
3 & -0.58 & 3 & $3.0 \pm 1.5$ & 0.9 & 0.39 & 4.5 & 0.59 \\
4 & +20.00 & 0 & $3.0 \pm 1.5$ & 0.9 & 0.24 & 6.1 & 0.77 \\
All & +2.05 & 12 & $10.4 \pm 3.0$ & 1.0 & - & 5.0 & 0.70 \\
\hline
\end{tabular}

Notes. Columns 3 and 4 show the number of events and expected number of background events within a $5^{\circ}$ distance from the assumed position of Cyg X-3 and within the shifted window boundaries. $n_{s}$ is the best-fit number of signal events from the likelihood maximization.

${ }^{a}$ The flux limits are given as $\Phi_{v_{\mu}}^{90 \%}$ in units of $10^{-11} \mathrm{TeV}^{-1} \mathrm{~cm}^{-2} \mathrm{~s}^{-1}$ which is the $90 \%$ C.L. upper limit on the differential muon neutrino flux such that $d \Phi_{v_{\mu}} / d E \leqslant \Phi_{v_{\mu}}^{90 \%} \cdot(E / \mathrm{TeV})^{-\gamma}, \gamma=3$ or 2 , respectively. $p$-Values above 0.5 are given as "-.".

$\Theta=2^{\circ}$ using a representative $E^{-2.6}$ power-law model under the assumption of a point-like neutrino signal located anywhere in the region. That is, assuming that the spatial correlation of signal events in the region is given only by the PSF of the analysis. The upper limits from the Cygnus region are at the level of $3 \times 10^{-11} \mathrm{TeV}^{-1} \mathrm{~cm}^{-2} \mathrm{~s}^{-1}$ for an $E^{-2.6}$ spectrum.

\subsection{Cygnus $X-3$}

In the flare search analysis of Cygnus X-3, no evidence for a signal is found in the neutrino sample for any of the sliding search windows. The smallest pre-trial $p$-value is $22 \%$ (resulting from the search with window 1). After correction for the trials, we arrive at a probability of $\approx 57 \%$ that this observation occurs in a background-only sample (final $p$-value). Upper limits on neutrino emission from Cyg X-3 during 20 days before and after the time windows have been determined using the method proposed by Feldman \& Cousins (1998) and are given in Table 4. Figure 17 shows the neutrino events close to Cyg X-3 as a function of time.

\section{CONCLUSIONS}

In this paper, we have presented dedicated searches for highenergy neutrino emission in the Galaxy. These analyses have been performed on data collected with two partial configurations of the IceCube neutrino telescope operating in conjunction with its predecessor AMANDA. In the two data-taking periods considered here, IceCube operated in a 22 string and in a 40 string configuration, and AMANDA was an integrated part of IceCube in both seasons. Results from several searches have been presented. We have performed a scan of the Galactic plane with the aim of discovering point-like neutrino emission in the part of the Milky Way that is located in the Northern Hemisphere. Since no significant local clustering of events has been observed, upper limits for soft-spectra neutrino emission from the Galaxy have been reported.

A search that is sensitive for many possible morphologies of neutrino emission, including, for example, the presence of several weak or extended sources, has been performed for the Cygnus region of the Galactic plane, yielding restrictive upper limits. Both a strong $\mathrm{TeV}$ gamma-ray source (MGRO 
J2019+37) and a TeV diffuse component have been measured in the Cygnus region with the Milagro detector from the area defined by Galactic latitude $-3^{\circ}<b<3^{\circ}$ and Galactic longitude $65^{\circ}<\ell<85^{\circ}$ (Abdo et al. 2007). The diffuse flux has been measured by Milagro over a region of $\approx 0.02$ sr and the total gamma-ray flux (diffuse and MGRO J2019+37) measured by Milagro accounts for $\approx 10^{-11} \mathrm{TeV} \mathrm{cm}^{-2} \mathrm{~s}^{-1}$ at $12 \mathrm{TeV}$ assuming a differential source spectrum of $E^{-2.6}$. Under the hypothesis that the region is transparent to gamma rays, the Milagro measurements can be used to estimate the maximal, associated neutrino flux (Kappes et al. 2007; Kelner et al. 2006). Assuming that all the high-energy gamma-rays reported by Milagro come from decays of $\pi^{0}$ produced in proton-proton interactions, and using the same $E^{-2.6}$ spectrum adopted in Abdo et al. (2007), the upper limits derived from the IC40+A analysis are only a factor of $\approx 2$ (Kappes et al. 2007; Kelner et al. 2006) above this estimate of the maximal neutrino flux from inside the Cygnus region. This implies that IceCube has the potential to detect neutrinos or to constrain the nature of the gamma-ray emission in one of the most active parts of the Galaxy in the next few years. Finally, a dedicated time-optimized search from the direction of the binary system Cygnus X-3 has been performed based on multi-wavelength observations. Upper limits for neutrino emission during specific episodes of enhanced radio and X-ray activity have been determined for this binary system.

We have presented the first neutrino point source searches that use a more densely instrumented sub-array inside a large neutrino telescope. The capability of improving the performance in the energy range below $\sim 10 \mathrm{TeV}$ in this way has been demonstrated. Using this capability, we have for the first time optimized a search for neutrino point sources particularly for the more steeply falling energy spectra expected for Galactic neutrino sources.

AMANDA, the sub-array used in this work, was decommissioned in 2009 and is now succeeded by IceCube-DeepCore (Abbasi et al. 2012b), an advanced low-energy extension of IceCube. The searches presented here have demonstrated that it is possible to improve the sensitivity to Galactic sources with early energy cutoffs and steeper spectra than $E^{-2}$ using a denser core array even at the expense of a larger atmospheric neutrino background. As a detector specifically built to enhance the sensitivity of IceCube at low energies, DeepCore is positioned in the deep center of the detector where the ice is clearest. This also allows us to use the outer strings of IceCube as an atmospheric muon veto and thus to go beyond the approach taken in this work to improve the sensitivity in the energy range below $\sim 10 \mathrm{TeV}$.

We acknowledge support from the following agencies: the U.S. National Science Foundation-Office of Polar Programs, the U.S. National Science Foundation-Physics Division, University of Wisconsin Alumni Research Foundation, the Grid Laboratory of Wisconsin (GLOW) grid infrastructure at the University of Wisconsin-Madison, the Open Science Grid (OSG) infrastructure; the U.S. Department of Energy, and National Energy Research Scientific Computing Center, the Louisiana Optical Network Initiative (LONI) grid computing resources; the National Science and Engineering Research Council of Canada; the Swedish Research Council, Swedish Polar Research Secretariat, Swedish National Infrastructure for Computing (SNIC), and Knut and Alice Wallenberg Foundation, Sweden; the German Ministry for Education and Research (BMBF), Helmholtz Al- liance for Astroparticle Physics (HAP), Deutsche Forschungsgemeinschaft (DFG), Research Department of Plasmas with Complex Interactions (Bochum), Germany; the Fund for Scientific Research (FNRS-FWO), FWO Odysseus programme, Flanders Institute to encourage scientific and technological research in industry (IWT), Belgian Federal Science Policy Office (Belspo); the University of Oxford, UK; the Marsden Fund, New Zealand; the Australian Research Council; the Japan Society for the Promotion of Science (JSPS); and the Swiss National Science Foundation (SNSF), Switzerland.

\section{REFERENCES}

Abbasi, R., Abdou, Y., Abu-Zayyad, T., et al. (IceCube Collaboration) 2010, NIMPA, 618, 139

Abbasi, R., Abdou, Y., Abu-Zayyad, T., et al. (IceCube Collaboration) 2011a, ApJ, 732, 18

Abbasi, R., Abdou, Y., Abu-Zayyad, T., et al. (IceCube Collaboration) 2011b, ApJ, 748, 118

Abbasi, R., Abdou, Y., Abu-Zayyad, T., et al. (IceCube Collaboration) 2011c, ApJ, 745, 45

Abbasi, R., Abdou, Y., Abu-Zayyad, T., et al. (IceCube Collaboration) 2011d, PhRvD, 84, 082001

Abbasi, R., Abdou, Y., Abu-Zayyad, T., et al. (IceCube Collaboration) 2012a, PhRvD, 85, 042002

Abbasi, R., Abdou, Y., Abu-Zayyad, T., et al. (IceCube Collaboration) 2012b, $\mathrm{APh}, 35,615$

Abbasi, R., Abdou, Y., Ackermann, M., et al. (IceCube Collaboration) 2009a, ApJL, 701, 47

Abbasi, R., Ackermann, M., Adams, J., et al. (IceCube Collaboration) 2009b, NIMPA, 601, 294

Abbasi, R., Ackermann, M., Adams, J., et al. (IceCube Collaboration) 2009c, PhRvD, 79, 062001

Abdo, A. A., Ackermann, M., Ajello, M., et al. (Fermi-LAT Collaboration) 2009a, Sci, 326, 1512

Abdo, A. A., Ackermann, M., Ajello, M., et al. (Fermi-LAT Collaboration) 2009b, ApJL, 701, 123

Abdo, A. A., Ackermann, M., Ajello, M., et al. (Fermi LAT Collaboration) 2009c, ApJL, 706, 1

Abdo, A. A., Allen, B., Berley, D., et al. 2007, ApJL, 658, 33

Ackermann, M. 2006, PhD thesis, Humboldt Universität zu Berlin

Ackermann, M., Ahrens, J., Bai, X., et al. (AMANDA Collaboration) 2006, NIMPA, 556, 169

Aharonian, F. A., Akhperjanian, A. G., Anton, G., et al. 2009, A\&A, 503, 817

Aharonian, F. A., Akhperjanian, A. G., Beilicke, M., et al. (HEGRA Collaboration) 2004, ApJ, 614, 897

Aharonian, F. A., Akhperjanian, A. G., Beilicke, M., et al. (HEGRA Collaboration) 2005, A\&A, 439, 635

Aharonian, F. A., Akhperjanian, A. G., Bazer-Bachi, A. R., et al. (HESS Collaboration) 2006a, A\&A, 457, 899

Aharonian, F. A., Akhperjanian, A. G., Bazer-Bachi, A. R., et al. (HESS Collaboration) 2006b, A\&A, 448, L43

Aharonian, F. A., Akhperjanian, A. G., Bazer-Bachi, A. R., et al. (HESS Collaboration) 2007, A\&A, 467, 1075

Aharonian, F. A., Krawczynski, H., Puehlhofer, G., \& Rowell, G. P. (HEGRA Collaboration) 2000, BAAS, 32, 1239

Ahrens, J. (The IceCube Collaboration) 2004, NIMPA, 524, 169

Albert, J., Aliu, E., Anderhub, H., et al. (MAGIC Collaboration) 2007a, A\&A, 474, 937

Albert, J., Aliu, E., Anderhub, H., et al. (MAGIC Collaboration) 2007b, ApJL, 664,87

Albert, J., Aliu, E., Anderhub, H., et al. (MAGIC Collaboration) 2007c, A\&A, 474, 937

Albert, J., Aliu, E., Anderhub, H., et al. (MAGIC Collaboration) 2007d, ApJL, 664, 87

Aleksić, J., Antonelli, L. A., Antoranz, P., et al. (MAGIC Collaboration) 2010, ApJ, 721, 843

Aleksić, J., Alvarez, E. A., Antonelli, L. A., et al. (MAGIC Collaboration) 2012, A\&A, 541, A13

Aliu, E. for the VERITAS Collaboration 32nd ICRC, Beijing 2011

Anchordoqui, L., Halzen, F., Montaruli, T., \& O'Murchadha, A. 2006, PhRvD, 76, 067301

Barthelmy, S. D., Barbier, L. M., Cummings, J. R., et al. 2005, SSRv, 120, 143

Bartoli, B., Bernardini, P., Bi, X. J., et al. 2012, ApJL, 745, 22 
Bednarek, W. 2005, ApJ, 631, 466

Barr, G. D., Gaisser, T. K., Lipari, P., Robbins, S., \& Stanev, T. 2004, PhRvD, 70, 023006

Begelman, M. C., Hatchett, S. P., McKee, C. F., Sarazin, C. L., \& Arons, J. 1980, ApJ, 238, 722

Beacom, J. F., \& Kistler, M. D. 2007, PhRvD, 75, 083001

Bell, A. R. 1978a, MNRAS, 182, 147

Bell, A. R. 1978b, MNRAS, 182, 443

Biermann, P. L., Becker, J. K., Meli, A., et al. 2009, PhRvL, 103, 061101

Biermann, P. L., Becker, J. K., Caceres, G., et al. 2010, ApJL, 710, 53

Borione, A., Catanese, M. A., Chantell, M. C., et al. 1998, ApJ, 493, 175

Braun, J., Baker, M., Dumm, J., et al. 2010, APh, 33, 175

Braun, J., Dumm, J., de Palma, F., et al. 2008, APh, 29, 299

Churchwell, E., Babler, B. L., Meade, M. R., et al. 2009, PASP, 121, 213

Conrad, J., Botner, O., Hallgren, A., \& Pérez de Los Heros, C. 2003, PhRvD, 67, 012002

Corbel, S. Fermi-LAT Collaboration 2010, BAAS, 42, 692

Efron, G. 1979, AnSta, 7, 1

Feldman, G., \& Cousins, R. 1998, PhRvD, 57, 3873

Fermi, E. 1949, PhRv, 75, 1169

Fermi, E. 1954, ApJ, 119, 1

Fesen, R. A. 1984, ApJ, 281, 658

Fiasson, et al. (H.E.S.S. Collaboration) 2009, in Proc. 31st Int. Cosmic Ray Conf., ed. J. Szabelski \& M. Giller (Kódź: Univ. Kódź), 0889

Finley, C. B., \& Westerhoff, S. 2004, APh, 21, 359

Frail, D. A., \& Hjellming, R. M. 1991, AJ, 101, 2126

Fry, J. N., \& Peebles, P. J. E. 1978, ApJ, 221, 19

Gallo, E., Fender, R., Kaiser, C., et al. 2005, Natur, 436, 819

Hayashi, S., Kajino, F., Naito, T., et al. 2009, APh, 32, 112

Hester, J. J. 2008, ARA\&A, 46, 127

Hill, G. C. 2003, PhRvD, 67, 118101

Hillas, A. M. 2005, JPhG, 31, 95

The High Resolution Fly's Eye (HIRES) Collaboration 2004, NuPhB, 136, 46

Jogler, T., Bosch-Ramon, V., Cortina, J., et al. 2008, JPhCS, 120, 062019

Kappes, A., Halzen, F., \& Murchadha, A. Ó. 2009, NIMPA A, 602, 117

Kappes, A., Hinton, J., Stegmann, C., \& Aharonian, F. A. 2007, ApJ, 656,870

Kelner, S. R., \& Aharonian, F. A. 2008, PhRvD, 78, 034013

Kelner, S. R., Aharonian, F. A., \& Bugayov, V. V. 2006, PhRvD, 74, 034018

Kennel, C. F., \& Coroniti, F. V. 1984, ApJ, 283, 710

Kerscher, M., Szapudi, I., \& Szalay, A. S. 2000, ApJ, 535, 13

Koljonen, K., Hannikainen, D., McCollough, M., et al. 2010, MNRAS, 406, 307

Kundu, M. R., \& Velusamy, T. 1967, AnAP, 30, 59

Landy, S. D., \& Szalay, A. S. 1998, ApJ, 494, 41

Le Duigou, J.-M., \& Knödlseder, J. 2002, A\&A, 392, 869
Levine, A. M., Bradt, H., Cui, W., et al. 1996, ApJL, 469, 33

Lozinskaya, T. A., Pravdikova, V. V., \& Finoguenov, A. V. 2002, AstL, 28, 223

Maddox, S. J., Efstathiou, G., \& Sutherland, W. J. 1996, MNRAS, 283, 1227

Marcowith, A., Komin, N., Gallant, Y. A., et al. 2008, in Proc. 30th Int. Cosmic Ray Conf., ed. R. Caballero et al. (Vol. 2; Mexico City: Universidad Nacional Autónoma de México), 787

Margon, B. 1984, ARA\&A, 22, 507

Meli, A., Becker, J. K., \& Quenby, J. J. 2008, A\&A, 492, 323

Migliari, S., Fender, R., \& Méndez, M. 2002, Sci, 297, 1673

Moskalenko, I. V., Porter, T. A., \& Strong, A. W. 2006, ApJL, 640, 155

Neunhöffer, T. 2006, APh, 25, 220

Neyman, J., \& Pearson, E. 1933, RSPT, 231, 289

Ohm, S., Fernandes, M. V., de Oña Wilhelmi, E., et al. 2010, in AIP Conf. Proc. 1381, 25th Texas Symp. on Relativistic Astrophysics, ed. F. M. Rieger, C. van Eldik, \& W. Hofmann (New York: Springer-Verlag), 257

Peebles, P. J. E. 1980, The Large Scale Structure of the Universe (Princeton, NJ: Princeton Univ. Press)

Peebles, P. J. E., \& Groth, E. J. 1975, ApJ, 196, 1

Pooley, G. G., \& Fender, R. P. 1997, MNRAS, 292, 925

Reed, J. E., Hester, J. J., Fabian, A. C., \& Winkler, P. F. 1995, ApJ, 440, 706

Reipurth, B., \& Schneider, N. 2008, in Handbook of Star Forming Regions, Vol. I, ed. B. Reipurth (The Northern Sky ASP Monograph Publications, Vol. 4; San Francisco, CA: ASP), 36

Reynoso, M. M., Romero, G. E., \& Christiansen, H. R. 2008, MNRAS, 387, 1745

Romney, J. D., Schilizzi, R. T., Fejes, I., \& Spencer, R. E. 1987, ApJ, 321, 822

Schlickeiser, R. 1989a, ApJ, 336, 243

Schlickeiser, R. 1989b, ApJ, 336, 264

Schneider, N., Bontemps, S., Simon, R., et al. 2006, A\&A, 458, 855

Sierpowska-Bartosik, A., \& Torres, D. F. 2009, ApJ, 693, 1462

Szostek, A., Zdziarski, A. A., \& McCollough, M. L. 2008, MNRAS, 388, 1001

Tavani, M., Bulgarelli, A., Piano, G., et al. (AGILE Collaboration) 2009, Natur, 462,620

Tavani, M., Bulgarelli, A., Piano, G., et al. (AGILE Collaboration) 2009, Natur, 462,620

Trimble, V. 1968, AJ, 73, 535

Trushkin, S. A., Bursov, N. N., Kotani, T., et al. 2007, in 6th Integral Workshop, The Obscured Universe, ed. S. Grebenev, R. Sunyaev, \& C. Winkler (ESA SP-622; Noordwijk: ESA), 357

Trushkin, S. A., Nizhelskij, N. N., \& Sotnikova, J. V. 2008, ATel, 1483

Tudose, V., Fender, R. P., Garrett, M. A., et al. 2007, MNRAS, 375, L11

van Eijndhoven, N., Fadiran, O., \& Japaridze, G. 2007, APh, 28, 456

Zornoza, J.-D.-D., \& Chirkin, D. 2008, in Proc. 30th Int. Cosmic Ray Conf., ed. R. Caballero et al. (Vol. 5; Mexico City: Universidad Nacional Autónoma de México), 1275

Zwart, J., Barker, R., Biddulph, P., et al. 2008, MNRAS, 391, 1545 